\title{
Neurofilaments Are Transported Rapidly But Intermittently in Axons: Implications for Slow Axonal Transport
}

\author{
Subhojit Roy, ${ }^{1}$ Pilar Coffee, ${ }^{2}$ George Smith, ${ }^{3}$ Ronald K. H. Liem, ${ }^{4}$ Scott T. Brady, ${ }^{2}$ and Mark M. Black ${ }^{1}$ \\ 1Department of Anatomy and Cell Biology, Temple University Medical School, Philadelphia, Pennsylvania 19140, \\ 2Department of Cell Biology, University of Texas Southwestern Medical Center, Dallas, Texas 75235, ${ }^{3}$ Department of \\ Physiology, University of Kentucky, Lexington, Kentucky 40536, and ${ }^{4}$ Department of Pathology, Columbia University \\ College of Physicians and Surgeons, New York, New York 10032
}

\begin{abstract}
Slow axonal transport conveys cytoskeletal proteins from cell body to axon tip. This transport provides the axon with the architectural elements that are required to generate and maintain its elongate shape and also generates forces within the axon that are necessary for axon growth and navigation. The mechanisms of cytoskeletal transport in axons are unknown. One hypothesis states that cytoskeletal proteins are transported within the axon as polymers. We tested this hypothesis by visualizing individual cytoskeletal polymers in living axons and determining whether they undergo vectorial movement. We focused on neurofilaments in axons of cultured sympathetic neurons because individual neurofilaments in these axons can be visualized by optical microscopy. Cultured sympathetic neurons were infected with recombinant adenovirus containing a construct encoding a fusion protein combining green fluorescent protein (GFP) with the heavy neurofilament protein subunit (NFH). The chimeric GFP-NFH
\end{abstract}

coassembled with endogenous neurofilaments. Time lapse imaging revealed that individual GFP-NFH-labeled neurofilaments undergo vigorous vectorial transport in the axon in both anterograde and retrograde directions but with a strong anterograde bias. NF transport in both directions exhibited a broad spectrum of rates with averages of $\approx 0.6-0.7 \mu \mathrm{m} / \mathrm{sec}$. However, movement was intermittent, with individual neurofilaments pausing during their transit within the axon. Some NFs either moved or paused for the most of the time they were observed, whereas others were intermediate in behavior. On average, neurofilaments spend at most $20 \%$ of the time moving and rest of the time paused. These results establish that the slow axonal transport machinery conveys neurofilaments.

Key words: neurofilaments; neurofilament proteins; axonal transport; green fluorescent protein; quantitative digital image analysis; cultured sympathetic neurons
Slow axonal transport is the mechanism whereby cytoskeletal proteins that are synthesized in the cell body are transported into the axon toward the axon tip. Slow axonal transport provides the axon with the architectural elements that are required to generate and maintain its elongate shape (Baas and Ahmad, 1993; Ahmad et al., 1994, 1998, 2000). The mechanisms of cytoskeletal transport have been the subject of heated debate. An early hypothesis based on the movement of pulse-labeled proteins in axonal transport proposed that cytoskeletal proteins were transported within the axon as polymers (Lasek, 1980; Tytell et al., 1981). Initially, the hypothesis stated that cytoskeletal polymers move down the axon en masse; however, this later was revised to propose that polymers moved more or less independently of each other (for review, see Baas and Brown, 1997). This hypothesis was satisfying intellectually because it could account for many of the known properties of the axonal cytoskeleton. However, attempts to test this hypothesis produced mixed results; some studies provided support for polymer transport (Baas and Ahmad, 1993; Ahmad and Baas, 1995; Yu et al., 1996; Slaughter et al., 1997), whereas others were used to argue that cytoskeletal polymers do not move in axons (Lim et al., 1990;

\footnotetext{
Received April 21, 2000; revised June 16, 2000; accepted June 20, 2000.

This work was supported by National Institutes of Health Grants NS17681 and NS34809 (M.M.B); National Institute of Neurological Disease and Stroke Grants NS23868 and NS23320, National Institute of Aging Grant AG12646, NASA Grant NAG2-962, and the Welch Foundation, number 1237 (S.T.B.); and National Institutes of Health Grant NS15182 (R.K.H.L.). Subhojit Roy was supported by a graduate fellowship from Temple University. We thank Dr. Virginia Lee for providing antibodies against the low-molecular-weight neurofilament subunit and Dr. Anthony Brown for providing software for tracking moving objects in time lapse sequences and also for sharing data before publication. We thank Dr. Gyorgyi Szebenyi for her work in characterizing the adenovirus vector and Theresa Slaughter for assistance in all phases of cell culturing.

Correspondence should be addressed to Dr. Mark M. Black, Department of Anatomy and Cell Biology, Temple University School of Medicine, 3400 North Broad Street, Philadelphia, PA 19140. E-mail: mblack@thunder.ocis.temple.edu. Copyright (C) 2000 Society for Neuroscience $0270-6474 / 00 / 206849-13 \$ 15.00 / 0$
}

Okabe and Hirokawa, 1990; Okabe et al., 1993; Sabry et al., 1995; Takeda et al., 1995; Chang et al., 1999). The lack of resolution of this issue can be attributed in part to the fact that most studies did not examine directly the movement of individual polymers in living axons. For example, studies arguing in favor of polymer transport did not observe movement directly but, instead, inferred movement on the basis of the effects of various experimental manipulations on polymer distribution. On the other hand, most studies that argued against polymer transport examined the behavior of populations of polymers in living axons. In general, these studies failed to detect movement of the population as a whole. However, movement of individual polymers could not be resolved in these analyses, especially if such movements occurred infrequently during the times that were used to evaluate whether the polymers move.

The importance of focusing on individual cytoskeletal polymers recently has been demonstrated by Wang et al. (2000). They tagged neurofilaments (NFs) so that they could be visualized microscopically in living neurons and directly observed the transport of NFs in growing axons. The movement was surprisingly rapid but interrupted by prolonged pauses. As a result, movement events were relatively infrequent, and at any moment in time most NFs were paused in their transit within the axon. As discussed by Wang et al. (2000), methods that focus on NF populations are not well suited to detect this movement.

We also have been studying NF transport in living axons and independently chose methods and systems similar to those used by Wang et al. (2000). We used cultured sympathetic neurons as a model system because the axonal NF array is sparse, permitting visualization of individual NFs by optical microscopy. NFs are heteropolymers consisting of three subunits termed NFL, NFM, and NFH for low-, middle-, and high-molecular-weight NF subunits (Ching and Liem, 1993; Lee et al., 1993). We labeled NFs in cultured sympathetic neurons by adenovirus transfection with a construct encoding green fluorescent protein (GFP) linked to 
NFH. The expressed chimeric GFP-NFH assembled into NFs that were visualized easily in living axons with epifluorescence optics. Observation of individual tagged NFs over time revealed that they undergo vigorous vectorial movements within axons in anterograde and retrograde directions and that transport in both directions exhibits a broad spectrum of rates. Our results confirm and expand on those of Wang et al. (2000) and unequivocally establish that cytoskeletal polymers are transported actively in axons and that the observed transport behavior of NFs can explain the behavior of neurofilament proteins as revealed by classic pulse chase studies on slow axonal transport.

\section{MATERIALS AND METHODS}

Materials. Culture media were obtained from Life Technologies (Grand Island, NY). Supplements for culture media were obtained from Life Technologies or Sigma (St. Louis, MO), except for nerve growth factor (NGF), which was purified from mouse salivary glands according to Mobley et al. (1976). Other reagents were obtained from Sigma unless otherwise indicated.

Adenovirus vectors. The tetracycline-inducible expression system used for these experiments consisted of two individual adenoviruses, one that contained the Tet-On transcriptional activator and the other that contained the tet-responsive element (TRE). To generate the Tet-On adenovirus, we excised pTet-On cDNA (Clontech, Palo Alto, CA) by using the restriction sites XhoI/NaeI and subcloned the pTet-On cDNA into the XhoI/XbaI sites of pXCJL.2 (gift from Dr. F. Graham, McMaster University, Ontario, Canada). To generate the adenovirus shuttle vector containing the TRE inducible promoter, we excised the TRE region from pTRE (Clontech), using $X h o \mathrm{I} / B a m \mathrm{HI}$, and subcloned it into the MluI/BamHI site of pXCRVS (Smith et al., 1996) to replace the RSV promoter.

A rat NFH cDNA (Chin and Liem, 1990) was subcloned into the pEGFP-C1 vector from Clontech, using restriction sites for SmaI and Bam HI to create a recombinant fusion protein with eGFP at the $\mathrm{N}$ terminus of NFH. The plasmid was amplified in Escherichia coli JM110 cells obtained from Stratagene (La Jolla, CA). After amplification the eGFP-NFH insert was subcloned into the adenovirus TRE adenovirus shuttle vector in two steps. First, GFP-NFH was cut out of pEGFP-C1 plasmid by using the restriction sites for AvrII and NheI and the GFPNFH fragment subcloned with part of the poly $\left(\mathrm{A}^{+}\right)$signal sequence into the SpeI site of pBluescript for amplification. The GFP-NFH [missing the poly $\left(\mathrm{A}^{+}\right)$fragment] then was cut out of pBluescript and subcloned into pXCTRE by using Bam HI.

To generate recombinant adenoviruses containing specific cDNA inserts, we cotransfected equimolar concentrations of either Tet-On or TRE shuttle vectors with pBGH11ts, a vector that encodes most of the viral genome needed to allow viral packaging, into 293 cells by using a liposomal transfection method (DOTAP), according to the manufacturer's protocol (Boehringer Mannheim, Indianapolis, IN). The liposomes were added to the wells of three 24 -well plates in which the 293 cells had reached $\approx 70 \%$ confluency. Monolayers of the 293 cells were incubated at $37^{\circ} \mathrm{C}$ overnight, refed, and incubated at $32^{\circ} \mathrm{C}$. Successful recombinations produced infectious virions that induced lysis of the 293 cells. This cytopathic effect (CPE) was monitored visually until total cell lysis in the well occurred. Viral DNA isolated from 100 to $200 \mu \mathrm{l}$ of supernatant from the wells displaying CPE was examined by PCR to determine whether virions contained the cDNA of interest (Zhang et al., 1993). Virus containing DNA inserts was plaque-purified twice and grown on 293 cells to produce large amounts of adenovirus. Plaque-purified viruses were examined to verify that no replication-competent virus contaminated the stocks. Tissue culture supernatant concentration for GFP-NFH/adenovirus averaged $2.5 \times 10^{8}$ plaque-forming units $(\mathrm{pfu} / \mathrm{ml})$. Tissue culture supernatant containing Tet-On adenovirus was concentrated further by centrifugation over cesium chloride via the method of Graham and Prevec (1991). The final concentration of Tet-On/adenovirus was $5.4 \times 10^{10} \mathrm{pfu} / \mathrm{ml}$.

Cell culture. Dissociated cultures of rat sympathetic neurons were prepared by using modifications of our previously published procedures (Brown et al., 1992; Li and Black, 1996) that were designed to maintain a $\mathrm{pH}$ of 7.3 in air. Culture dishes were prepared by drilling a $10-\mathrm{mm}$ diameter hole through the bottom of each dish and then fixing an acidwashed glass coverslip (no. 1 thickness) to the bottom of the dish with paraffin. Then the coverslips were coated with poly-L-lysine plus laminin on the surface facing the interior of the culture dish, as described previously (Brown et al., 1992). Neurons dissociated from superior cervical ganglia of 1- to 3-d-old rat pups by using sequential treatments with collagenase and trypsin, followed by trituration (Black et al., 1996), then were plated onto the substrate-coated glass coverslips in L15-based media as described by Chun and Patterson (1977), except that methylcellulose was not used and 3\% fetal calf serum was used instead of rat serum. Under these conditions the neurons rapidly attach to the substrate and begin extending axons during the next several hours. By the next morning most neurons have elaborated one or more axons that are several hundred microns in length.

Beginning on the day after plating the neurons were incubated with an equimolar mix of Tet-on and TRE-GFP-NFH adenoviruses that were used at a final concentration of $3-5 \times 10^{6} \mathrm{pfu} / \mathrm{ml}$ each for $\approx 24 \mathrm{hr}$. Then the medium was replaced with virus-free medium containing tetracycline $(0.2-1 \mathrm{mg} / \mathrm{ml})$ to stimulate expression of the transfected GFP-NFH chimera. On the basis of visual inspection of the GFP-NFH fluorescence, transfection was readily detectable beginning the day after the addition of tetracycline and continued for several days. Expression level within cultures was variable, with some cells exhibiting little or no fluorescence, some fluorescing very brightly, and others exhibiting modest levels of fluorescence. Frequently, we observed that cells exhibiting very bright GFP-NFH fluorescence had stunted axons, presumably caused by superinfection with multiple TRE-GFP-NFH adenoviruses. However, neurons exhibiting moderate or low GFP-NFH fluorescence had normalappearing axons in terms of overall length and branching patterns. In the present experiments the neurons were imaged at 2-4 d after inducing the expression of GFP-NFH. Only cells exhibiting moderate levels of fluorescence were selected for analysis.

Live cell imaging. On the day of imaging the culture medium was removed, and $\approx 200 \mu \mathrm{l}$ of fresh medium containing oxyrase (diluted 1:100 from the stock supplied by Oxyrase, Mansfield, $\mathrm{OH}$ ) was placed onto the surface of the coverslip containing the cells. This volume of medium slightly overfilled the small well over the coverslip formed by the hole in the bottom of the culture dish and served as a reservoir of medium during observation. Then the well was sealed by placing a sterile coverslip over it to minimize gaseous exchange between the surrounding air and the culture medium and to prevent evaporation. The neurons were incubated for at least $2 \mathrm{hr}$ before imaging.

To examine the movement of GFP-NFH containing NFs, we placed culture dishes on the stage of a Zeiss Axiovert 135 inverted microscope equipped with epifluorescent optics (see below for details). The use of the L15-based medium ensured that medium $\mathrm{pH}$ was maintained at 7.3. To control temperature on the stage, we enclosed the entire microscope in a custom-designed Plexiglas box into which warm air was circulated from a Nicolson air stream stage incubator to maintain a temperature of $35 \pm 1^{\circ} \mathrm{C}$. The objective also was heated to $36^{\circ} \mathrm{C}$ with a Bioptechs objective heater (Butler, PA). Under these conditions the neurons continued to extend axons at normal rates for at least $6 \mathrm{hr}$, the longest time that was examined. Neurons were observed by epifluorescence microscopy with $100 \times / 1.3$ numerical aperture Plan Neofluar oil immersion or $63 \times / 1.4$ numerical aperture Plan Apochromatic oil immersion objectives (Zeiss, Thornwood, NY). Neurons were illuminated with a $100 \mathrm{~W}$ mercury arc lamp, observed with filter sets obtained from Chroma (Brattleboro, VT), and optimized for GFP (filter set 41014) or rhodamine (filter set 41002). A heat-absorbing colored glass filter (BG40) was inserted into the light path between the light source and the filter block. The intensity of light was controlled electronically by an Atto Arc mercury bulb power supply (Potomac, MD). For live cell imaging the illumination intensity typically was set at $25 \%$ of maximum. Maximum light intensity was used to image the fixed cells.

Images were captured with a Princeton Instruments cooled CCD camera (Roper Scientific, Princeton, NJ) equipped with a Princeton Instruments $1000 \times 800$ back-thinned CCD chip. Image acquisition was performed with Apple Macintosh G3 or G4 computers that used IP Lab software (Scanalytics, Fairfax, VA) to control the camera. Illumination of the sample was controlled with a Uniblitz electronic shutter (Vincent Associates, Rochester, NY), which was operated automatically from the IP Lab software with a MAC 2000 Communications Interface Module (Ludl Electronic Products, Hawthorne, NY). Images were acquired with user-defined regions of interest (the maximum usable area of the CCD chip measured $1000 \times 800$ pixels, and each pixel was $15 \mu \mathrm{m}^{2}$ ) and were stored in full 12-bit format. Before capturing a series of images, we saved an instantaneous readout of the bias voltage offset on the chip and subsequently subtracted it from each exposed image. The magnification of the CCD images was calibrated with a stage micrometer. For presentation the images were scaled to eight-bits, saved in TIFF format, and then imported into Adobe Photoshop to compose the figures; text and arrowheads were added with Adobe Illustrator. Colorized versions of the gray scale images that were obtained with the cooled CCD camera were prepared with Adobe Photoshop.

Cell extraction and fixation. Unextracted cells were fixed by immersion in cold $\left(-20^{\circ} \mathrm{C}\right)$ methanol for $10 \mathrm{~min}$. Fixed cells were rehydrated with PBS and then stained as described below. In some experiments the neurons were extracted before fixation. All steps were performed at room temperature. Cultures were rinsed once with PBS and once with PHEM [containing (in mM) 60 PIPES, 25 HEPES, 10 EGTA, and $2 \mathrm{MgCl}_{2}$, pH 6.9 (Schliwa and van Blerkom, 1981)] and then were extracted for 10 min with PHEM containing $0.02 \%$ saponin plus $0.2 \mathrm{M} \mathrm{NaCl}$. Extracted cells were fixed by immersion in cold methanol as described above. These extraction plus fixation conditions cause a loosening of the axonal NF array so that individual NFs separate from each other for variable distances along their length and thus can be imaged by immunofluorescence procedures (Brown, 1997).

Immunofluorescence procedures. To examine the overall distribution of NFs, we double-stained neurons that were fixed without extraction with a polyclonal antibody against NFL to reveal NFs (generously provided by Dr. Virginia Lee, Department of Pathology, University of Pennsylvania) and with an antibody to tyrosinated tubulin (yl1/2; Accurate Scientific, Westbury, New York) to reveal overall cell morphology. Cells were incubated with blocking solution (PBS containing $10 \mathrm{mg} / \mathrm{ml} \mathrm{BSA}$ and $10 \%$ normal donkey serum) for $15 \mathrm{~min}$ just before incubation with primary 
antibodies and again before incubation with secondary antibodies [lissamine-rhodamine-labeled donkey anti-rabbit and cy5-labeled donkey anti-mouse secondary antibodies (AffiniPure grade, Jackson ImmunoResearch, West Grove, PA)]. In addition, all antibodies were diluted in blocking solution and then clarified before use by centrifugation at $200,000 \times g$ for 10 min in a Beckman TL-100 ultracentrifuge. Cells were incubated simultaneously with the anti-NFL and anti-tubulin antibodies that were diluted 1:500 and 1:100, respectively, for $45 \mathrm{~min}$ at $37^{\circ} \mathrm{C}$. After extensive rinsing the cells were incubated with both secondary antibodies, each diluted 1:100, for $30 \mathrm{~min}$ at $37^{\circ} \mathrm{C}$, rinsed extensively with $\mathrm{PBS}$, and then mounted in $50 \%(\mathrm{w} / \mathrm{v})$ glycerol/PBS containing $10 \mathrm{mg} / \mathrm{ml}$ of $n$-propyl gallate. Transfected cells expressing GFP-NFH that were extracted to cause NF splaying were stained with the NFL antibody as described above and were mounted in $50 \%(\mathrm{w} / \mathrm{v})$ glycerol/PBS containing $10 \mathrm{mg} / \mathrm{ml}$ of $n$-propyl gallate. Then the cells were imaged to compare the localization of GFP-NFH with NFs.

Analysis of NF transport in living neurons. At the time of examination most neurons had multiple axons that branched repeatedly, and individua axons were much too long to be contained in an individual microscope field. As a result, only a relatively small portion of the axon could be examined during a single imaging session. In general, we focused attention on regions in the middle portion of the axon, although more limited analyses of NF movements in the proximal axon or in growth cones also were performed. Neurons expressing GFP-NFH were identified visually by using the criteria indicated above and positioned on the microscope stage so that a portion of their axonal arbor was in the field of view. We selected regions that contained clear discontinuities or gaps in the GFPNFH fluorescence. Then, by using macros prepared in IP Lab, we obtained time lapse sequences. In general, exposures were of 1-2 sec duration, and images were acquired every $5 \mathrm{sec}$. Total imaging time varied but was a maximum of 20 min for a single imaging session.

Quantitative analyses of transport were performed with the Oncor Imaging software package, using a customized program designed to track individual objects over time. The program was created by Dr. Anthony Brown (Department of Biological Sciences, Ohio University) and generously was provided to us. We used this program to track the position of the leading or trailing ends of moving GFP-NFH-containing structures from one frame to the next in our image sequences. The program recorded the coordinates of the tracked object and then computed the distance moved $/ 5$ sec interval and transport rate $/ 5 \mathrm{sec}$ interval. We tracked only objects for which the leading or trailing ends could be identified unambiguously from one frame to the next in the image sequences. Statistical analyses were performed with StatView software (Abacus Concepts, Berkeley, CA), using the unpaired Student's $t$ test and the Mann-Whitney nonparametric test (because similar results were obtained with both tests, data are presented on the basis of Student's $t$ test in most cases).

\section{RESULTS}

Cytoskeletal proteins synthesized in the neuron cell body are delivered to the axon by the process of slow axonal transport. Although the process itself is well documented, the mechanisms are unknown. One issue of long-standing controversy is whether cytoskeletal proteins are transported as assembled polymers or in some other form. In the present studies we show unequivocally that NFs are transported in living axons of cultured neurons, and we describe many features of NF transport behavior. We used cultured sympathetic neurons for these experiments and tagged NFs for light microscopic visualization by transfecting the neurons with a construct that encoded an assembly-competent GFP-NFH chimera. The culture system proved to be essential to the success of these experiments because the NF array in the axons of these neurons is relatively sparse, thereby permitting visualization of individual NFs containing GFP-NFH in living axons. First, we describe essential features of the NF array in cultured sympathetic neurons and the use of the GFP-NFH construct to label NFs in these neurons. Then we document transport behavior of NFs in living axons.

\section{The neurofilament array in axons of cultured rat sympathetic neurons is discontinuous}

Figure 1 shows fluorescent images of portions of axons that were fixed without extraction and then stained for NFs and tubulin. Tubulin staining is continuous along the axon, whereas NF staining is clearly discontinuous. Specifically, there are regions within the axon that stain well for NFs interspersed with regions that do not stain at all. Wang et al. (2000) reported similar discontinuities in the NF array in axons of cultured sympathetic neurons. We refer to regions devoid of NF staining as gaps. The length of gaps is quite variable, ranging from a few microns to several tens of microns. In

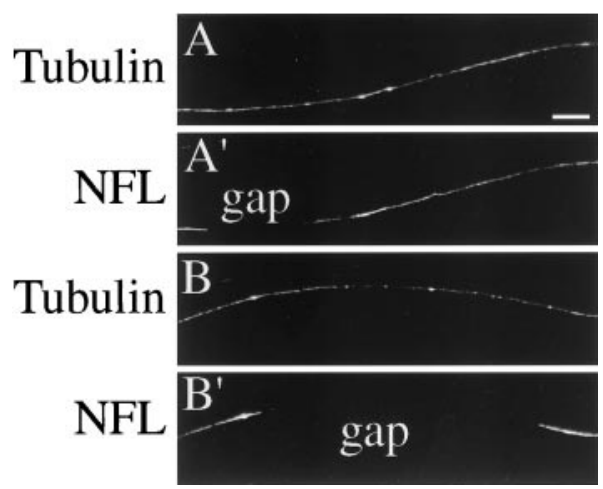

Figure 1. The neurofilament array is discontinuous along the length of the axon. Neurons were fixed without extraction and then double-stained as described in Materials and Methods to reveal tyrosinated tubulin and NFL. Portions of two axons are shown. Tyrosinated tubulin staining is continuous along the axon $(A, B)$, whereas NFL staining is discontinuous, with obvious gaps in which no detectable staining for NF is apparent $\left(A^{\prime}, B^{\prime}\right)$. Scale bar, $8.3 \mu \mathrm{m}$

addition, as discussed below, gaps in the axonal $\mathrm{NF}$ array also can be seen in living neurons transfected with GFP-NFH. As viewed in living neurons, the gaps are dynamic, changing their appearance and dimensions as NFs bordering the gaps move (see, for example, Figs. 4,5 ). The frequency of gaps is also variable, with some axons having few gaps, whereas other axons have many. We do not have the sense that gaps occur in particular regions of the axon, except that generally they are not present in the proximal $\approx 50 \mu \mathrm{m}$ of the axon. This region of the axon tends to be relatively thick and stains all along its length for NFs. In contrast, in more distal regions the axon are much thinner, and it is in these thinner regions that gaps in the NF array typically were observed. In our transport studies we focused on gaps in the NF array and asked whether NFs in regions adjacent to the gaps moved into and through them, as predicted by the slow transport model involving asynchronous movement of cytoskeletal polymers (Lasek et al., 1992, 1993).

\section{GFP-NFH is assembly-competent and reliably labels NF in axons}

To label NFs in living axons for microscopic visualization and transport studies, we transfected cultured neurons with a construct that encoded an inducible GFP-NFH chimera. The transfection protocol and general features of expression are described in $\mathrm{Ma}$ terials and Methods. Here we address the use of this approach to tag axonal NFs. selectively

Several observations demonstrate the assembly competence of transfected GFP-NFH. First, in transfected neurons fixed and stained for NFs by using an antibody against NFL, individual NFs frequently could be seen in the cell body by NFL staining, and GFP-NFH colocalized precisely with these NFs (Fig. 2). Second, to determine whether the GFP-NFH in axons was associated specifically with NFs, we extracted and fixed transfected neurons via conditions that cause individual NFs in the axon to separate from each other for variable distances along their length so that they can be imaged by immunofluorescence procedures (Brown, 1997). Such preparations then were stained with an antibody against NFL to reveal NFs, and we determined whether GFPNFH localized to axonal NFs. Figure 3 shows a portion of an axon that was processed in this manner and then imaged to reveal NFs and GFP-NFH fluorescence. Many NFs splayed out from axon shaft, and all of these NFs contained GFP-NFH. Typically, GFP$\mathrm{NFH}$ is distributed sporadically along the length of individual NFs. GFP-NFH association with NFs was detected all along the axon wherever NFs were seen. Finally, in non-neuronal cells (data not shown) GFP-NFH had a filamentous distribution that is expected for intermediate filaments in these cells. Collectively, these results demonstrate that the GFP-NFH is assembly-competent and associates with NFs in the neuronal cell body and axon. 

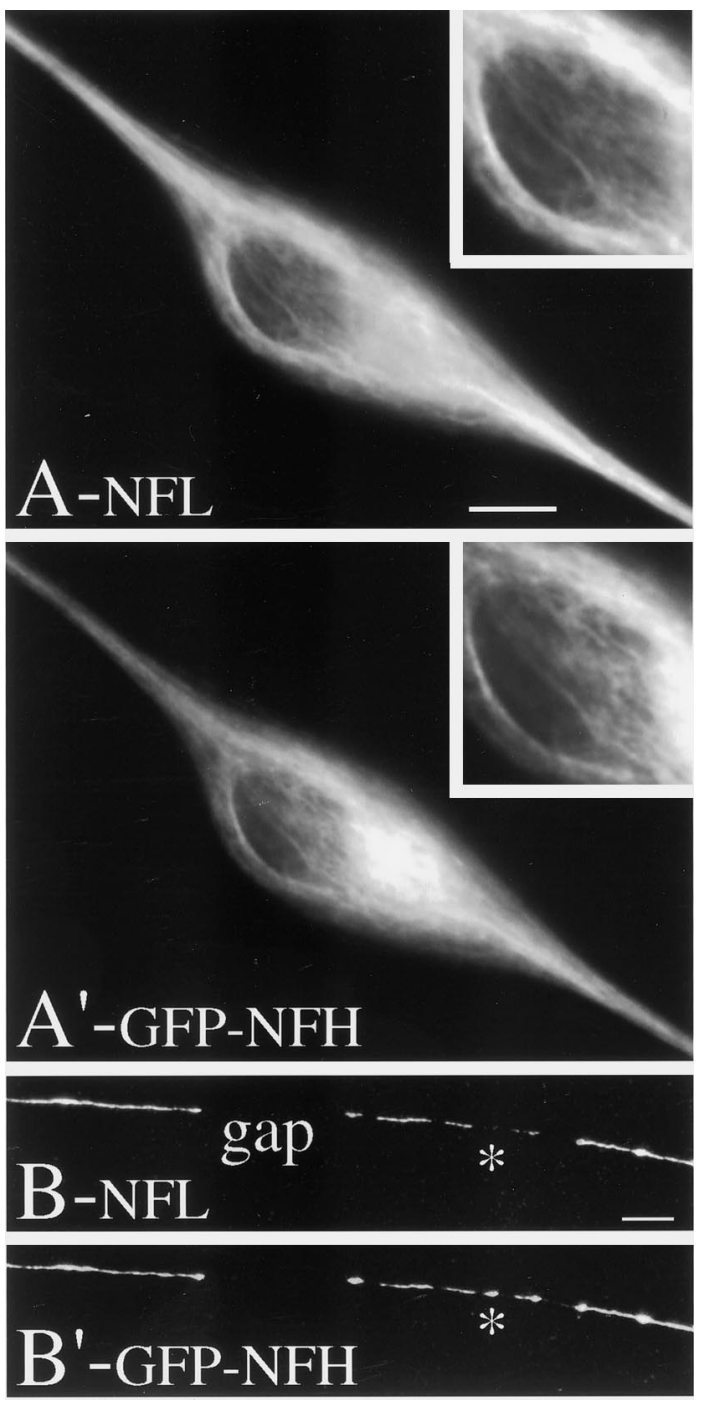

Figure 2. GFP-NFH colocalizes with NFL in cell bodies and axons. Neurons transfected with the GFP-NFH construct were fixed without extraction by immersion in cold methanol, stained with antibodies to NFL, and then imaged to reveal NFL staining $(A, B)$ and GFP-NFH $\left(A^{\prime}, B^{\prime}\right)$. $A$ and $A^{\prime}$ show a neuron cell body in which the focal plane is between the bottom of the nucleus and the bottom of the cell. The filamentous character of NFL is readily apparent in this region, and the GFP-NFH colocalizes with the filamentous structures stained by NF. In the insets the region beneath the nucleus is shown digitally zoomed. $B$ and $B^{\prime}$ show part of an axon of a transfected cell. The NFL staining is discontinuous in this region, with an obvious gap in NFL staining. The GFP-NFH localization mirrors the pattern seen by NFL staining in that the regions that stain for NFL also have GFP-NFH fluorescence, and the gaps in NFL staining also do not have detectable GFP-NFH fluorescence. The one exception is indicated by the asterisk, which highlights a region of dim NFL fluorescence but comparatively bright GFP-NFH fluorescence. See Results for further details. Scale bar, $8.3 \mu \mathrm{m}$.

Another important issue is whether the transfected GFP-NFH associates principally with NFs or whether there is a substantial pool of GFP-NFH that is unassembled and thus is soluble or in some other nonfilamentous form. The images of GFP-NFH and NFL localization in the cell body shown in Figure 2 support the view that the unassembled pool of GFP-NFH is at most a small portion of the assembled pool. For these images we focused specifically on the relatively thin cytoplasm between the bottom of the nucleus and bottom of the cell. We were able to obtain relatively sharp images of NFL staining and GFP-NFH fluorescence in this region because the nucleus that overlies it lacks NFs and NF proteins. The GFP-NFH colocalized precisely with the filamentous structures revealed by NFL staining, and, importantly, the intensity of GFP-NFH fluorescence associated with these struc- tures was much greater than that seen in adjacent regions that contained no NFs. In addition, we did not observe any discrete concentrations of GFP-NFH in regions that did not contain NFs. These observations indicate that, in the neuron cell body where the pool of unassembled GFP-NFH is expected to be greatest, most GFP-NFH fluorescence was associated with NFs, and none was detected in a structured form other than as NFs.

To address the issue of assembled versus unassembled GFPNFH specifically in axons, we took advantage of the following facts. First, the NF array is discontinuous (see above). Second, the vast majority of NFL in neurons is assembled into NFs. We previously have used biochemical procedures to document this for the same type of neurons used in the present studies (Black et al., 1986). This point is reinforced further by the observation that NFL staining along the axon reveals regions that stain well for NFL adjacent to regions that do not stain at all (see Figs. 1, 2; see also Wang et al., 2000). Such results are not consistent with the existence of a sizable pool of unassembled NFL in these axons. Third, under in vivo conditions the assembly of NFH requires the presence of NFL (Ching and Liem, 1993; Lee et al., 1993). Thus, if transfected GFP-NFH in the axon is principally or only assembled into NFs, then it will be found only in regions of the axon that stain for NFL, and it will not be detected in axonal regions that do not stain for NFL. On the other hand, if a sizable pool of unassembled GFP-NFH exists in axons, then axonal regions devoid of NFs should contain detectable GFP-NFH.

To test these predictions, we fixed transfected neurons without extraction, stained them for NFs with an antibody against NFL, and then imaged them to reveal NFL and GFP-NFH localization. Typical results are shown in Figure 2, $C$ and $D$. Discontinuities in NFL staining are readily apparent, with regions of strong staining interspersed with regions having no detectable staining. GFP$\mathrm{NFH}$ fluorescence almost exactly parallels the pattern seen by NFL staining. Specifically, regions that stain for NFL also have GFP-NFH fluorescence, whereas gaps that lack detectable NFL staining also do not have detectable GFP-NFH fluorescence. It is also important to note that gaps in GFP-NFH fluorescence are also readily apparent in living neurons (see Figs. 4, 5). The presence of such gaps is inconsistent with the existence of a sizable pool of unassembled GFP-NFH in axons of transfected neurons. Infrequently, we have seen GFP-NFH in regions that contain little or no NFL staining (asterisk in Fig. 2C,D). In this circumstance GFP-NFH typically had the appearance of small spherical or oblong structures. The significance of this observation is unclear, although these structures may correspond to vesicular structures containing NF and other cytoskeletal proteins (Hollenbeck and Bray, 1987; Hollenbeck, 1993; Chang et al., 1999). These latter structures were distinct in appearance and thus easily distinguished from the filamentous structures that stained for NFL and also contained GFP-NFH. Collectively, these results indicate that most GFP-NFH in axons is associated with NFs and that the pool of GFP-NFH that is unassembled or in other non-NF forms is negligible and thus does not interfere with the detection of NFs containing GFP-NFH.

\section{Visualization of NF transport in living axons}

In our transport studies we tagged axonal NF with the GFP-NFH chimera and then focused on gaps in the NF array to determine whether NFs containing GFP-NFH moved vectorially within the axon. We reasoned that the zero background of GFP-NFH in the gaps would make it possible to detect whether tagged NFs initially located outside of the gaps would be transported into and through the gaps. Our results unambiguously demonstrate the translocation of GFP-NFH-containing NFs in the axon. The transport occurred in both anterograde and retrograde directions, and NFs were translocated over distances of many tens of microns at rates that ranged from $\leq 0.1$ to $>2 \mu \mathrm{m} / \mathrm{sec}$. Figures 4 and 5 show representative examples of NFs undergoing transport in the anterograde or retrograde direction, respectively.

Figure 4 shows a portion of a sequence that demonstrates the 
NFL

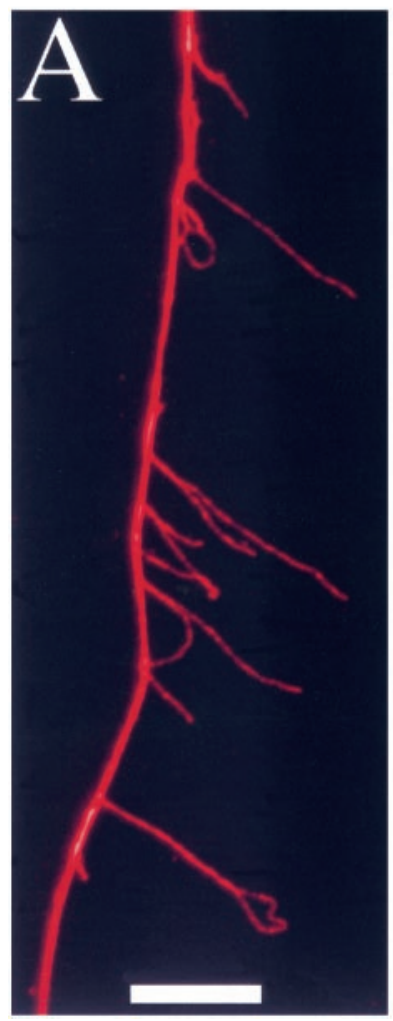

\section{GFP-NFH}

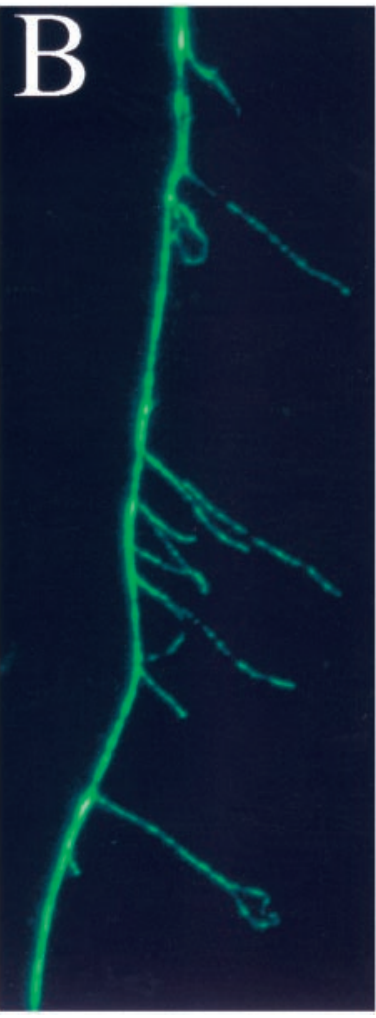

\section{Overlay}

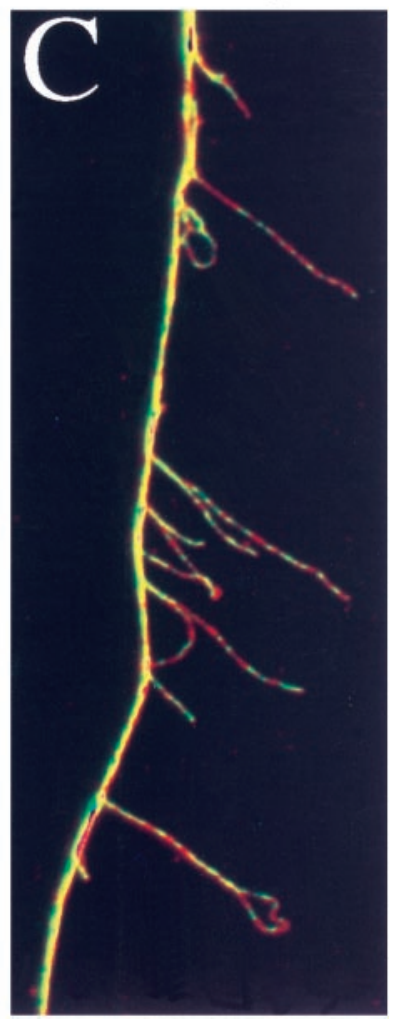

Figure 3. GFP-NFH is associated with axonal neurofilaments. Transfected neurons were extracted and then fixed by using conditions that cause axonal NFs to splay apart from each other. The resulting preparations were stained for NFs with an antibody against NFL and then imaged to reveal NFL staining and GFP-NFH. $A$ shows NFs as revealed by NFL staining, $B$ shows GFP-NFH, and $C$ is a color overlay showing that the GFP-NFH precisely localizes with NFs. Note that NFL staining along individual NFs frequently appears nonuniform, and GFP-NFH also appears nonuniformly associated with individual NFs. Frequently, regions of dim NFL staining along NFs correspond to especially bright fluorescence for GFP-NFH and vice versa. This accounts for the observation that, in the color overlay, individual NFs often appear as alternating patches of red and green. Presumably, the presence of GFP-NFH impairs the accessibility of the antiNFL antibody to its antigen, thus accounting for the dimmer NFL fluorescence at sites containing GFP$\mathrm{NFH}$ as compared with adjacent sites without GFP-NFH. Scale bar, $8.3 \mu \mathrm{m}$. transport of a GFP-NFH-labeled NF into and through a gap in the anterograde direction. The gap was $\approx 62 \mu \mathrm{m}$ long. The tagged NF first was detected in the gap at $395 \mathrm{sec}$ in the sequence and moved steadily through the gap during the next $95 \mathrm{sec}$, at which time it left the field of view. In the middle of the sequence the entire tagged $\mathrm{NF}$ was apparent and measured $\approx 14 \mu \mathrm{m}$ in length. The single arrowheads show the leading edge of the moving filament. Quantitative analyses of the change in position of the NF over time showed that it moved through the gap at an average rate of 0.79 $\mu \mathrm{m} / \mathrm{sec}$, although the transport rate was quite variable during the sequence, ranging from 0.18 to $1.23 \mu \mathrm{m} / \mathrm{sec}$.

Figure 5 shows a GFP-NFH-labeled NF that was transported retrogradely through a gap. In this sequence the gap is $\approx 35 \mu \mathrm{m}$ long, and two discrete GFP-NFH-containing objects move retrogradely through the gap (the single arrowheads identify the back edge of the trailing object). These two objects exhibit nearly identical transport behavior through the gap, and we suspect that they are different segments of the same NF. The trailing object is $6.7 \mu \mathrm{m}$ long, the leading object is $6.2 \mu \mathrm{m}$ long, and the space between them is $3.2 \mu \mathrm{m}$ long, for a combined length of $16.1 \mu \mathrm{m}$. This filament alternates between intervals of vectorial movement and pauses, when no detectable movement occurs. Such pauses are common for both retrogradely and anterogradely moving NFs (see below for more details; see also Wang et al., 2000). The average rate of transport of the retrogradely moving NF shown in Figure 5 during the entire time for which this object could be tracked, including pauses, is $0.33 \mu \mathrm{m} / \mathrm{sec}$; the average transport rate, excluding pauses (defined as a change in position of $<0.3 \mu \mathrm{m}$ in $5 \mathrm{sec}$; see below), is $0.47 \mu \mathrm{m} / \mathrm{sec}$, with a range from 0.06 to $0.96 \mu \mathrm{m} / \mathrm{sec}$. These findings confirm previous reports of retrograde transport of NFs in mature (Glass and Griffin, 1994) and cultured (Wang et al., 2000) neurons.

\section{Anterogradely and retrogradely transported NFs move at similar rates}

We have compared transport rates of anterogradely moving NFs with those of retrogradely moving NFs. For this purpose we wanted to compare transport rates when NFs actually were moving and not paused. To distinguish when NFs were moving versus paused, we defined movement as a change in position within the axon of $\geq 0.3 \mu \mathrm{m}$ in $5 \mathrm{sec}$. At $100 \times$ magnification this distance corresponds to two pixels with our camera and represents the lower limit of our ability to detect movement reliably. Similarly, a change in position of $<0.3 \mu \mathrm{m}$ in $5 \mathrm{sec}$ was defined as a pause. Figure $6 \mathrm{~A}$ shows frequency distributions of the average transport rates (excluding pauses) for all anterogradely and retrogradely moving NFs that were analyzed in the present studies. For each NF we computed its average rate of transport on the basis of all $5 \mathrm{sec}$ intervals of observation in which the NF met the criteria for movement (i.e., it moved $\geq 0.3 \mu \mathrm{m}$ in $5 \mathrm{sec}$ ). The resulting histograms, Figure $6 \mathrm{~A}$, reveal that both anterogradely and retrogradely transported NFs move at a broad spectrum of average transport rates. For anterogradely moving NFs $(n=52)$ the range of average rates (excluding pauses) was $0.14-1.7 \mu \mathrm{m} / \mathrm{sec}$, whereas for retrogradely moving NFs $(n=21)$ the range was $0.11-1.4 \mu \mathrm{m} / \mathrm{sec}$. Next, the distribution of transport rates for anterogradely moving NFs is very similar to that for retrograde moving NFs, and statistical analysis indicates that the two distributions are not significantly different from each other (unpaired $t$ test, $p>0.1$ ). Finally, we observed that the majority $(71 \%)$ of NFs that were observed to be moving were transported anterogradely. All of these observations agree well with those of Wang et al. (2000), who reported that $83 \%$ of the NFs that were observed moved anterogradely and that average anterograde transport rates ranged from 0.02 to $1.21 \mu \mathrm{m} / \mathrm{sec}$, whereas average retrograde movements ranged from 0.15 to $1.82 \mu \mathrm{m} / \mathrm{sec}$. We have estimated a combined rate of transport that includes all of the anterogradely and retrogradely moving NFs that were analyzed by assigning negative values to the retrogradely transported NFs and then computing the average of the rates for all NFs. This revealed a net anterograde transport of $0.3 \mu \mathrm{m} / \mathrm{sec}$. The bias toward the anterograde direction is consistent with observations at the macroscopic level that showed a net transport of NF proteins in the anterograde direction (Koehnle and Brown, 1999).

We also compared maximum transport rates for the population 

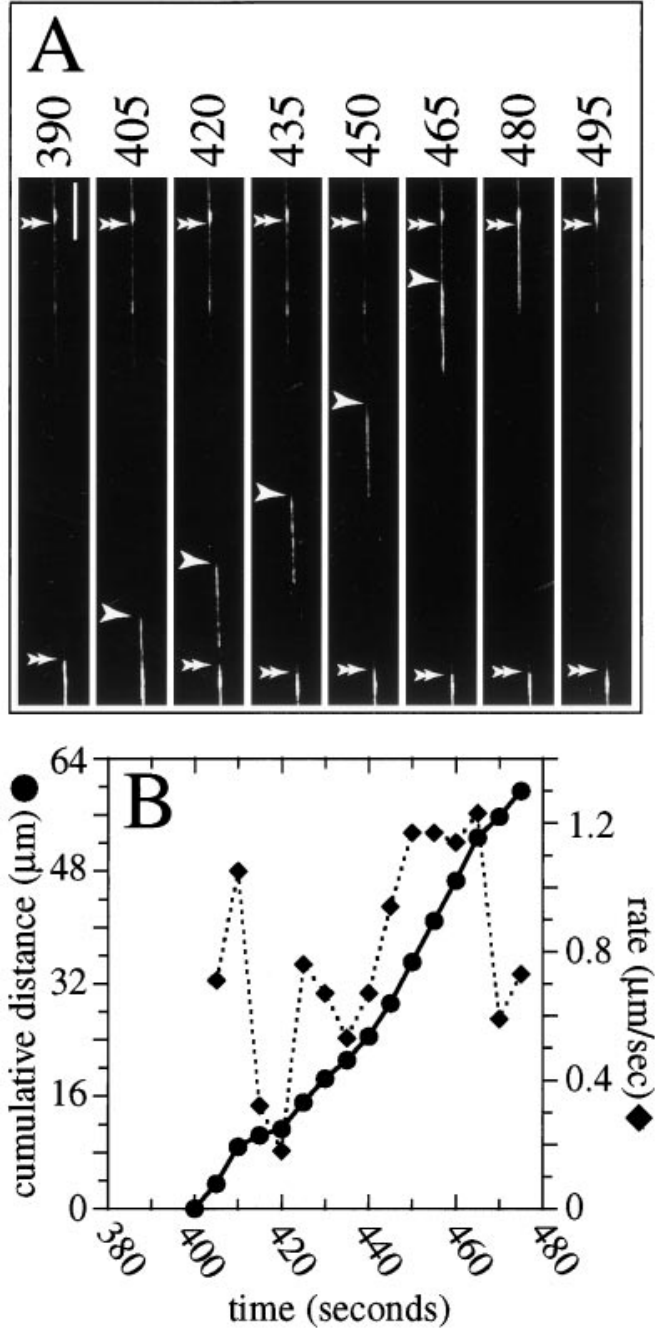

Figure 4. Anterograde transport of NFs. $A$ shows selected images from a time lapse series that depicts the movement of a GFP-NFH-labeled NF moving anterogradely (the cell body is down in the figure, and the axon tip is $u p)$. The numbers above each frame indicate the time in seconds. At the start of sequence a relatively large gap in the GFP-NFH fluorescence occupies most of the field. A NF moves into and through the gap during the time course of the sequence, first appearing a little earlier than $405 \mathrm{sec}$ and leaving the field by $495 \mathrm{sec}$. The single arrowheads indicate the front of the moving NF, whereas the double arrowheads indicate NFs that did not move during the sequence. Scale bar, $8.3 \mu \mathrm{m}$. $B$ shows the average transport rate of the NF for each $5 \mathrm{sec}$ interval of the sequence superimposed on the cumulative distance moved versus time. See supplementary information for a video of this sequence at http://thunder.ocis.temple.edu/ mblack.

of anterogradely and retrogradely moving NFs (Fig. 6B). Again, retrogradely and anterogradely transported NFs were indistinguishable in this comparison (unpaired $t$ test, $p>0.1$ ). Also, the maximum transport rate varied over a broad range. For anterogradely moving NFs the range was $0.33-2.2 \mu \mathrm{m} / \mathrm{sec}$, with a mean of $1.14 \mu \mathrm{m} / \mathrm{sec}(\mathrm{SD}=0.44)$. For retrogradely moving NFs the maximum transport rates varied from 0.2 to $3 \mu \mathrm{m} / \mathrm{sec}$, with a mean of $1.33 \mu \mathrm{m} / \mathrm{sec}(\mathrm{SD}=0.67)$.

We observed a few NFs that moved bidirectionally, exhibiting sustained transport in one direction followed by sustained movement in the opposite direction (see NF2 in Fig. 9). Such NFs provide a unique opportunity to compare anterograde and retrograde rates for single polymers, controlling for effects specific to a given structure. NF2 in Figure 9 is especially well suited for this comparison because it underwent several spurts of relatively sustained anterograde movement interspersed with sustained retrograde transport over a relatively long period of time. Figure 7 shows a comparison of anterograde and retrograde rates for this
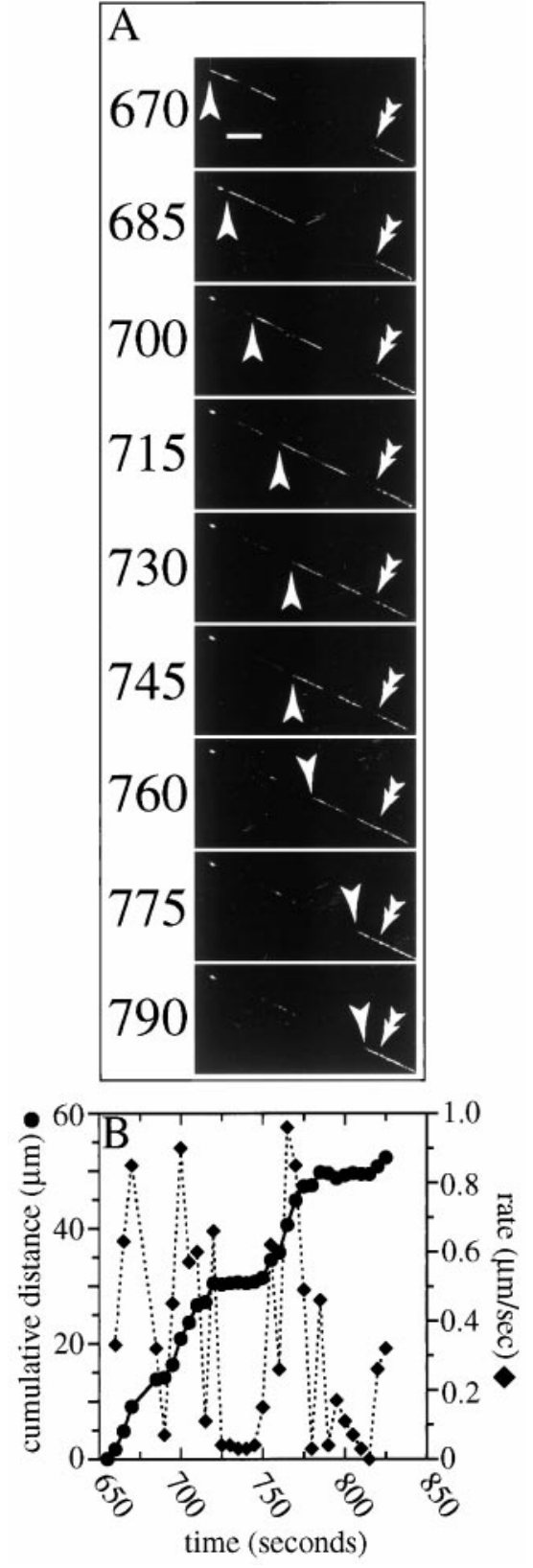

Figure 5. Retrograde transport of NFs. $A$ shows selected images from a time lapse series that depicts the movement of a GFP-NFH-labeled NF moving retrogradely (the cell body is down in the figure, and the axon tip is up). The numbers to the left of each frame indicate the time in seconds. At the start of the sequence a gap in the GFP-NFH fluorescence occupies the middle of the field. Two filamentous structures labeled with GFP-NFH move into and through the gap (as discussed in Results, it is likely that these two objects are different parts of the same NF). The single arrowheads indicate the trailing end of the NF, and the double arrowheads indicate NFs that did not move during the sequence. The moving NF first appears a little earlier than $670 \mathrm{sec}$ in the sequence (see $B$ ) and moves through the gap during the subsequent $\approx 120 \mathrm{sec}$. Note that, in the middle portion of the sequence, the NF appears to pause in its transit through the gap. Scale bar, $8.3 \mu \mathrm{m}$. $B$ shows the average transport rate of the NF for each 5 sec interval of the sequence superimposed on the cumulative distance moved versus time. See supplementary information for a video of this sequence at http://thunder.ocis.temple.edu/ mblack.

NF. For this analysis we compared the transport rates for each 5 sec interval that the NF moved in the anterograde direction with those for movements in the retrograde direction. Transport rates in the two directions were statistically indistinguishable from each other ( $p>0.1$, unpaired $t$ test). Similar results were obtained for four other NFs that were observed to undergo bidirectional move- 


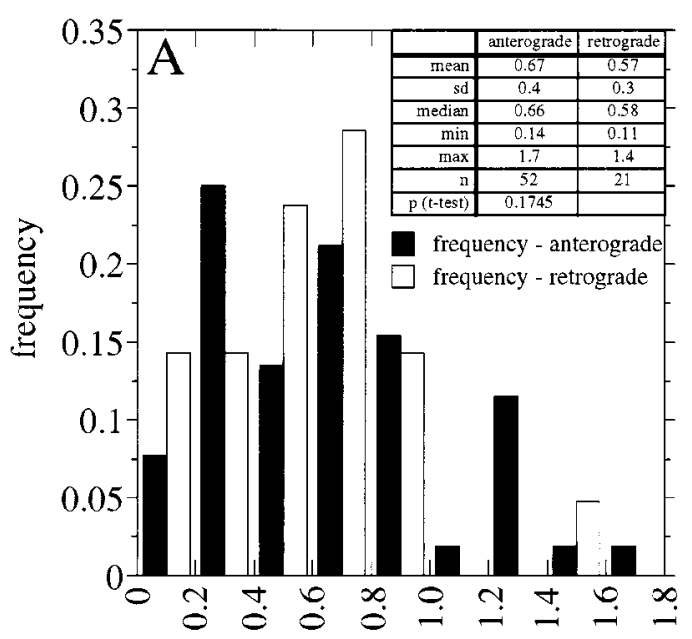

average rate $(\mu \mathrm{m} / \mathrm{sec})$

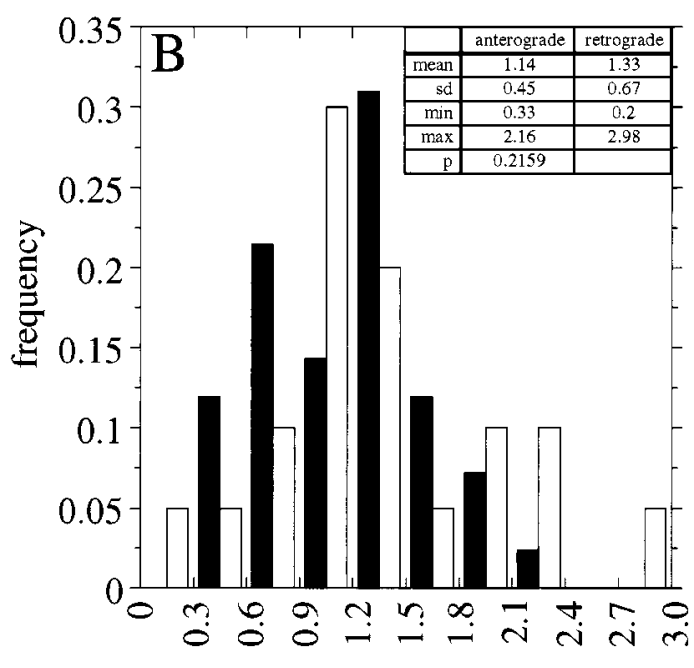

maximum rate $(\mu \mathrm{m} / \mathrm{sec})$

Figure 6. Comparison of transport rates of anterogradely and retrogradely moving NFs. $A$ shows frequency distributions of the average transport rate (excluding pauses) for anterogradely and retrogradely moving NFs, and $B$ shows maximum transport rates for anterogradely and retrogradely moving NFs. The average and maximum transport rates of each moving NF were calculated as described in Results. For the population of NFs that were examined, the average and maximum transport rates for anterogradely moving NFs were not statistically different from those for retrogradely moving NFs.

ment. Three of five NFs that exhibited clear bidirectional movement were transported in a net anterograde direction. Collectively, these results indicate that mechanisms that power anterograde and retrograde NF transport move polymers over a comparable range of transport rates.

\section{The transport of individual NFs occurs at a broad range of rates and is intermittent}

Population analyses of NF transport revealed a striking variability in transport rates for both anterograde and retrograde transport. A similar variability was seen in the transport of individual NFs (for example, see the NFs depicted in Figs. 4, 5 and data documented in greater detail for the four NFs shown in Figs. 8, 9). Several features of NF transport are noteworthy. First, transported NFs commonly exhibit intervals of relatively sustained transport interspersed with quiescent intervals in which they move much more slowly or not at all. These latter intervals appear as relatively flat portions of the graphs in the cumulative distance plots, whereas intervals of sustained movement may span many tens of seconds

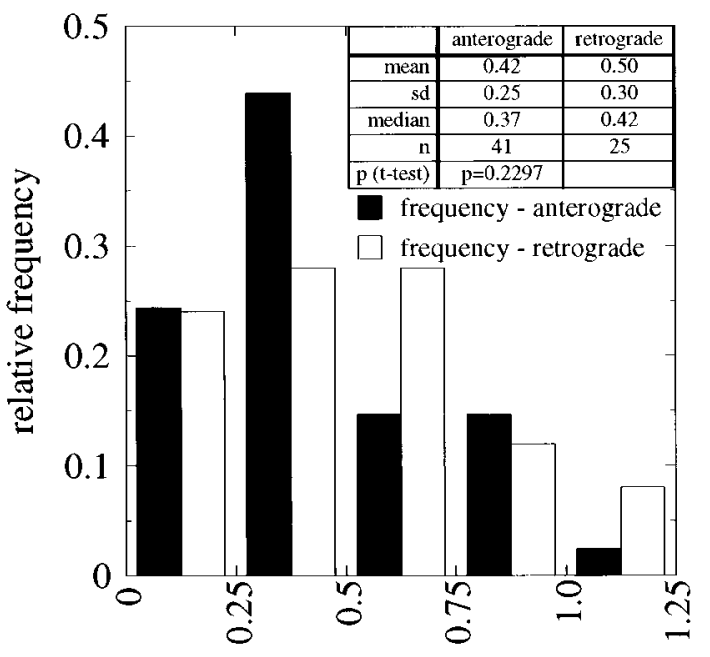

rate $(\mu \mathrm{m} / \mathrm{sec})$

Figure 7. Anterograde and retrograde transport rates of a NF that moved bidirectionally. NF2 in Figure 9 moved bidirectionally over a period of $\approx 400 \mathrm{sec}$, exhibiting spurts of sustained movement in one direction alternating with spurts in the opposite direction. We compared the transport rates for each $5 \mathrm{sec}$ interval that the NF moved anterogradely with the rates for each $5 \mathrm{sec}$ interval that it moved retrogradely. Shown is a frequency distribution of the anterograde and retrograde transport rates for this one $\mathrm{NF}$; these two sets of rates were statistically indistinguishable from each other.

and have a distinctly positive or negative slope corresponding to anterograde or retrograde movements, respectively. Although it is clear that these two intervals differ quantitatively in their average transport rates, detailed inspection reveals that each interval exhibits features common to the other. For example, within intervals of sustained movement the NFs often exhibit brief quiescent periods of relatively slow or no movement. Similarly, the quiescent intervals of overall slower movement often exhibit extended pauses in movement that are punctuated by brief intervals of movement, spanning 5-10 sec in our imaging sequences. During these brief intervals of NF movement the rates are often comparable to those seen during intervals of sustained movement. The principal difference between intervals of sustained movement and quiescent intervals may be in the relative proportion of time spent moving versus paused.

All of the above described features of NF transport can be seen for the NFs illustrated in Figure 8, which undergo sustained movement for an extended interval, move much more slowly for a subsequent interval, and then start moving more rapidly again. Quiescent intervals comprise clear pauses, in which NFs did not move detectably, interspersed with brief periods of movement. As a result, some vectorial movement may occur during quiescent intervals, but both the duration of movement and the distance moved are much less than those seen during intervals of sustained movement. This general type of behavior was relatively common and was exhibited by NFs moving in both anterograde and retrograde (see Fig. 5) directions. For any given NF the proportion of time spent moving versus pausing was quite variable. At the extremes, some NFs moved in a sustained manner for most or all of the time that they were observed (see Fig. 4), whereas others were paused for most or all of the observation time (see Fig. 10 and below). Other NFs were intermediate in behavior, spending substantial time moving and pausing. This group includes examples in which the majority of time was spent moving (see Fig. 5) or paused (see Fig. $8 A$ ) or split approximately equally between moving and paused (see NF1 in Fig. 9). As discussed in more detail below, at any moment in time most axonal NFs appeared to be paused, and only a small fraction of the total was actually moving.

Another feature of NF transport revealed by analyses of individual NFs is that a single NF may move at a broad range of rates. 

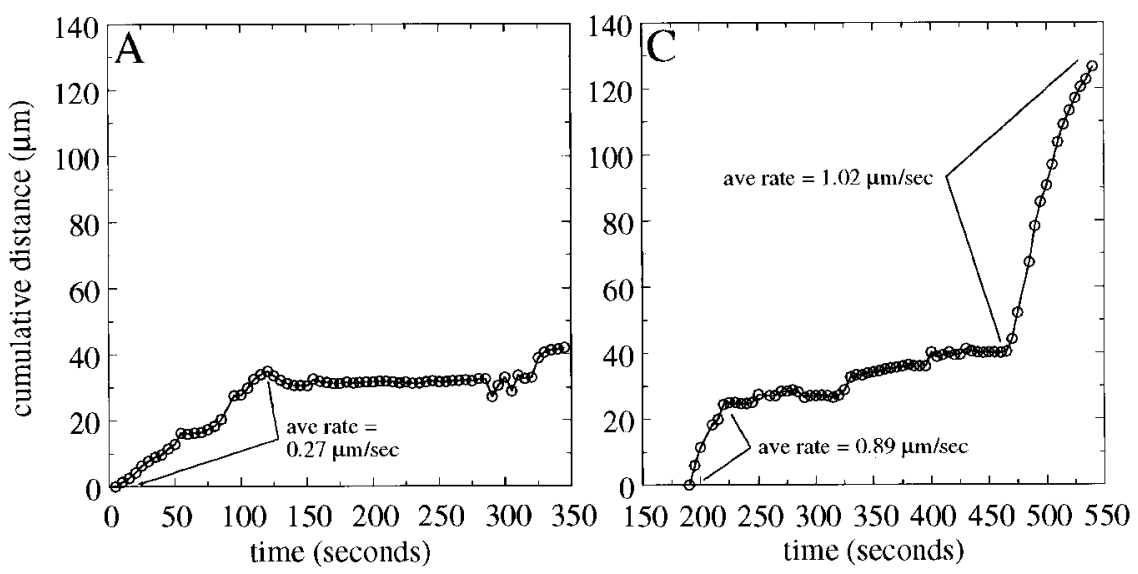

Figure 8. Quantitative analysis of the transport of individual NFs. Data for two representative NFs are shown, both exhibiting net anterograde movement. $A$ and $B$ show data from one NF; $C$ and $D$ show data from the other NF. $A$ and $C$ show cumulative distance plots for these NFs. Note that both NFs spend a portion of the time undergoing relatively sustained movement and a portion of the time moving much more slowly or not at all. $B$ and $D$ show frequency distributions of the transport rate of these NFs computed for each $5 \mathrm{sec}$ interval that they were observed; positive and negative values correspond to the rates for anterograde and retrograde movements. Both NFs exhibited a broad spectrum of transport rates during the times that they moved.
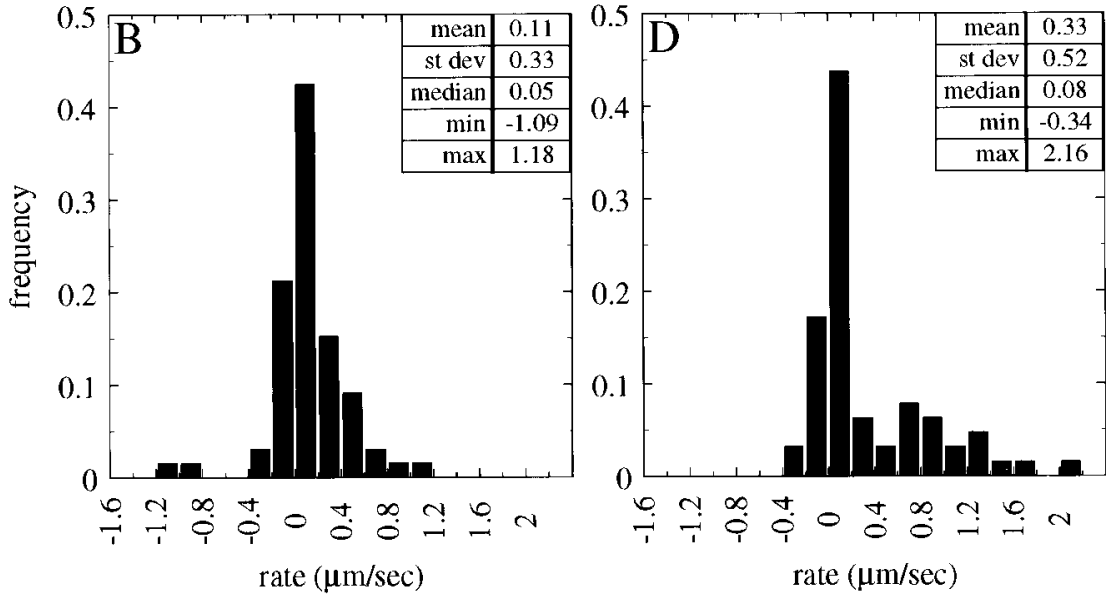

This point can be seen in histograms of Figures 8 and 9, which show that individual NFs may move at rates varying by a factor of fiveto 10 -fold or more during the time that they are observed. For example, the NF in Figure $8 C$ was monitored for a total of $350 \mathrm{sec}$. This NF exhibited two intervals of sustained movement separated by an extended interval of relative quiescence. We calculated a transport rate for this NF for every $5 \mathrm{sec}$ interval during which it was observed. During the entire time of observation this NF moved at rates ranging from 0 to $2.2 \mu \mathrm{m} / \mathrm{sec}$, whereas during periods of sustained movement the rates varied from 0.3 to 2.2 $\mu \mathrm{m} / \mathrm{sec}$ and during quiescent periods rates ranged from 0 to 0.8 $\mu \mathrm{m} / \mathrm{sec}$. Although the absolute range of transport rates that a given $\mathrm{NF}$ exhibited varied from one NF to another, most NFs moved at a variety of rates during their transit through the axon, regardless of their direction of transport.

\section{NFs move asynchronously during their transit in the axon}

The original description of NF transport suggested that NFs move en masse in a highly coordinated manner within the axon (Lasek and Hoffman, 1976; Black and Lasek, 1980). However, subsequent findings that NF proteins exhibited a broad spectrum of rates and that the NF array varies in structure along the axon (Lasek et al., 1992, 1993; Nixon, 1998) indicated that this view of NF transport was incorrect. Instead, NFs were proposed to move independently of each other during transport within the axon. The present findings confirm and expand on this latter view. First, individual NFs move at a range of rates that varies by more than an order of magnitude (see Fig. 6; see also Wang et al., 2000). Second, at any moment in time some NFs within an individual axon are moving while others are paused (see Figs. 4, 5, 10). Third, moving NFs in a single axon often exhibit very different behaviors. A particularly striking example of this is shown in Figure 9, which shows the behavior of two NFs moving within the same gap during the same period of time. One NF, NF1, underwent anterograde transport and exhibited two periods of sustained movement alternating with quiescent periods. The other NF, NF2, moved bidirectionally, displaying spurts of transport in the anterograde direction alternating with retrograde spurts. Clearly, the transport behavior of any given NF appears independent of others in the same axon. As a result, the NF population in an axon progresses down that axon in a highly asynchronous manner.

\section{Short and long NFs move at the same rate}

To test whether variations in NF length contribute to variability in transport behavior for different NFs, we compared transport rates of long NFs with those of short NFs. NF length was estimated for GFP-NFH-tagged NFs in which both ends of the labeled segment were clearly visible. We assumed that the length of the GFP-NFHlabeled segment corresponded to the length of the NF. One caveat for this assumption is that GFP-NFH did not label NFs continuously along their length (see Fig. 3). Thus, it is possible that substantial lengths of NFs remain unlabeled. This is unlikely, however, because in all of the splayed preparations that were examined, the discontinuities in NF labeling with GFP-NFH were relatively short, and estimates of NF length on the basis of staining with an NFL antibody corresponded closely to those based on GFP-NFH labeling (see Fig. 3; data not shown). Thus, the length of NF polymer containing GFP-NFH is expected to be a reasonable approximation of the length of the polymer itself.

Of the 73 transported NFs examined in this study, we were able to visualize both ends of 44 of them. These filaments ranged in length from 1.5 to $39 \mu \mathrm{m}$, with a mean $\pm \mathrm{SD}$ of $9.8 \pm 8.9$. Examples of transport behavior for long $(30.5 \mu \mathrm{m})$ and short $(3.7 \mu \mathrm{m}) \mathrm{NFs}$ are shown in Figure 8, $A$ and $C$, respectively. When moving, the shorter NF was transported at an average rate of $0.6 \mu \mathrm{m} / \mathrm{sec}$, whereas the longer NF moved at an average rate of $0.2 \mu \mathrm{m} / \mathrm{sec}$. Although this suggests that relatively short NFs move faster than relatively long NFs, more detailed analysis failed to confirm a consistent difference. Specifically, we compared the average trans- 

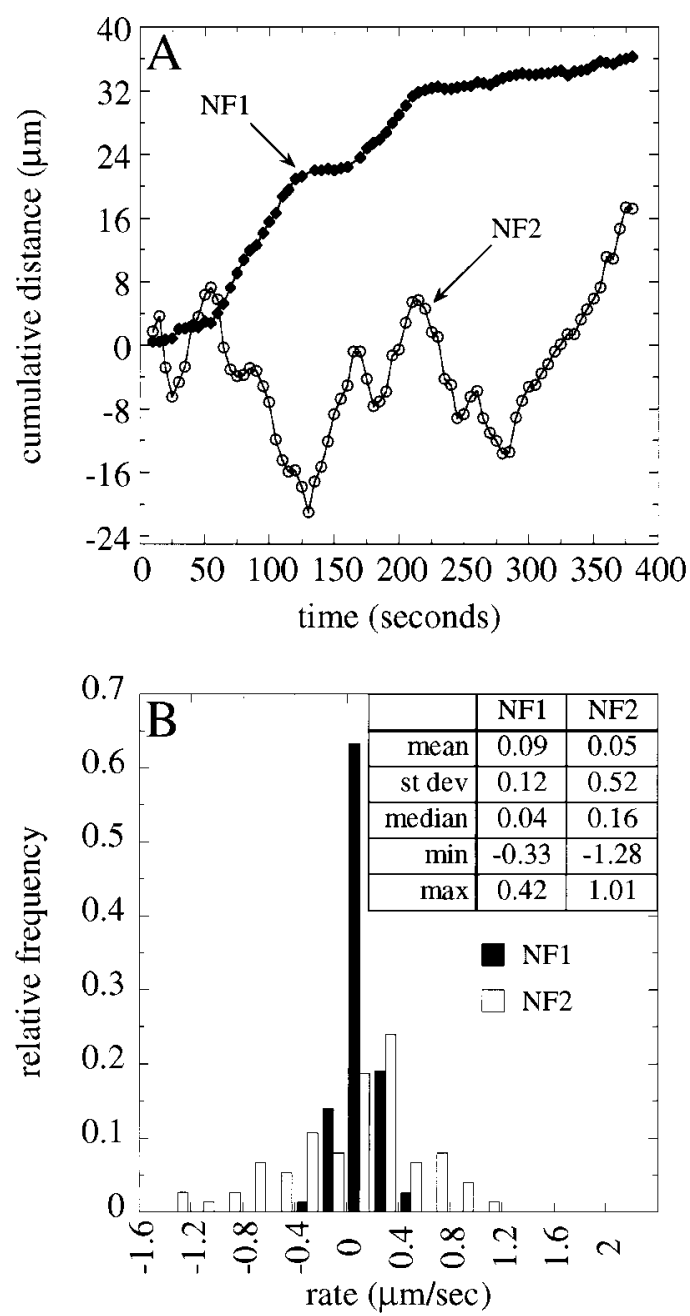

Figure 9. NF transport is highly asynchronous. Shown are cumulative distance plots $(A)$ and frequency distributions of transport rates $(B)$ for two NFs that moved in the same region of an axon during the same time interval. The two NFs are designated $N F 1$ and $N F 2$ in $A$; the frequency distributions of transport rates for $N F 1$ and $N F 2$ are shown in $B$ (filled and open bars, respectively). Note that, although these NFs are present in the same region of an axon, they exhibit very different transport behaviors. See supplementary information for a video of this sequence at http://thunder. ocis.temple.edu $/ \sim$ mblack.

port rates of NFs $\leq 5 \mu \mathrm{m}$ with those of NFs $\geq 30 \mu \mathrm{m}$. NFs $\leq 5 \mu \mathrm{m}$ long were transported at rates ranging from 0.2 to $1.4 \mu \mathrm{m} / \mathrm{sec}$, with an average of $0.7 \pm 0.4 \mu \mathrm{m} / \mathrm{sec}$ (mean $\pm \mathrm{SD}, n=13$ ), whereas $\mathrm{NFs} \geq 30 \mu \mathrm{m}$ long were transported at rates ranging from 0.2 to $1 \mu \mathrm{m} / \mathrm{sec}$, with an average of $0.5 \pm 0.3 \mu \mathrm{m} / \mathrm{sec}(n=7)$. These means were not statistically different from each other (unpaired $t$ test, $p>0.1$; Mann-Whitney test, $p>0.1$ ), indicating that transport rate apparently does not vary systematically within the range of NF lengths that were examined. We also compared shorter versus longer NFs in terms of their peak rates. The average peak rates for NFs $\leq 5 \mu \mathrm{m}$ long and NFs $\geq 30 \mu \mathrm{m}$ long were $1.3 \pm 0.7$ and $1.1 \pm 0.4 \mu \mathrm{m} / \mathrm{sec}$, respectively. These values were not statistically different from each other (unpaired $t$ test, $p>0.1$ ).

\section{At any moment in time many NFs are paused in their transport within the axon}

Although the preceding sections emphasized movement of NFs in axons, we also have noted that NFs pause during their transit within the axon and that the proportion of time spent moving versus pausing is quite variable from one NF to another. From observations of many sequences of GFP-NFH-labeled NFs in axons, the impression was that, at any given point in time, most NFs were not moving (see also Wang et al., 2000). For example, the

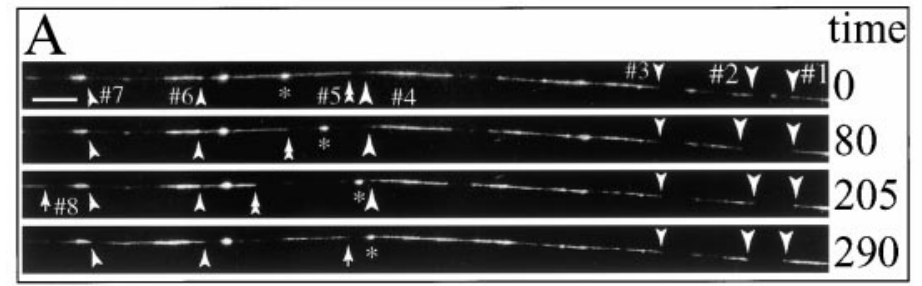

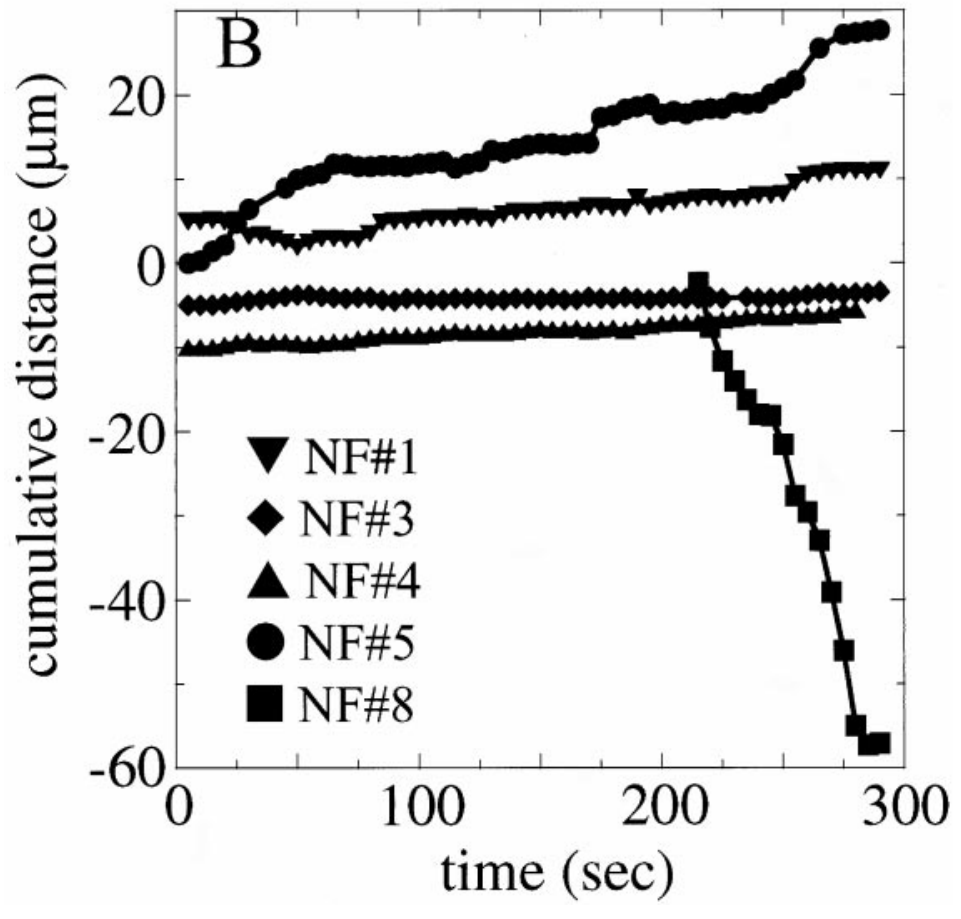

Figure 10. Behavior of multiple NFs in a single axon. A 146- $\mu$ m-long segment of an axon was imaged for $5 \mathrm{~min}$. This axon segment contains several gaps in the GFP-NFH fluorescence, thereby permitting detection of the ends of several NFs. $A$ shows selected frames from the sequence in which the ends of eight different NFs are indicated. Six of these $(1-4,6$, and 7) moved very little or not at all and are highlighted with single arrowheads. One (5) moved anterogradely; its trailing end is indicated with a double arrowhead. The remaining $\mathrm{NF}(8)$ moved retrogradely, and its leading edge is indicated with an arrow. See supplementary information for a video of this sequence. $B$ shows cumulative distance plots for five of these NFs, specifically those labeled $1,3,4,5$, and 8 . Note that NFs 1,3 , and 4 undergo very little net movement during the sequence and, in fact, spend most of the total time paused, undergoing no detectable movement (to minimize overlap of the data points for the plots for NFs 1, 3, and 4, we have shifted them on the $y$-axis). The anterogradely moving NF (5) shows clear intervals of movement but spends most of the time paused. The retrogradely moving NF (8) first appears in the sequence at $205 \mathrm{sec}$ and moves in a relatively sustained manner until the end of the sequence. The asterisk (in $A$ ) identifies an ovoid structure that contains GFP-NFH that moves in a net retrograde direction. This object is much brighter than individual NFs that contain GFP-NFH and is distinctly different in shape. Similar structures have been seen by Hollenbeck and Bray (1987) and Chang et al. (1999) and probably are associated with the endosomal/lysosomal pathway.

GFP-NFH-tagged NFs highlighted with double arrowheads in Figures 4 and 5 did not undergo transport during the time period that is shown. Sequences depicted in Figures 4 and 5 focus on gaps in the NF array to reveal NFs moving into and through the gaps. Although NFs that did not move are apparent in these sequences, the extent to which NFs are paused in their transit within the axon is underestimated. To provide a better sense of this issue, we imaged relatively long lengths of axons that had multiple gaps in the NF array for an extended time. This provided an opportunity to monitor many NFs over an extended interval. Figure 10 shows one such sequence in which moving and paused NFs are apparent. This sequence captures events in a 146- $\mu$ m-long segment of an axon over a time period of $\approx 5 \mathrm{~min}$. The ends of eight different NFs are apparent in this sequence. During this sequence two NFs can 
be seen undergoing relatively sustained vectorial movement, one in the anterograde direction and one in the retrograde direction. The other labeled NFs move very little or not at all. Sequences in which a majority of NFs move very little or not at all were common in our experience. Collectively, these findings gave the impression that, at any moment in time, only a minority of NFs in the axon is actually undergoing transport. The rest are either paused or show very slow movement. This view is reinforced by numerous examples of individual NFs that transiently paused in their translocation within the axon, often for extended periods of time (see Figs. 8, 9).

The sequence shown in Figure 10 affords a unique opportunity to quantify the relative amount of movement for several NFs in a single axon over a relatively extended time interval (i.e., $5 \mathrm{~min}$ ). One end of each of the eight NFs that could be identified unambiguously was tracked for as long as it could be followed in this sequence. The specific NFs that were tracked are indicated in the top panel of Figure 10, and the bottom panel shows cumulative distance plots for several of these NFs, including two that exhibited substantial movement. Of these latter NFs, the anterogradely moving NF (NF 5, filled circles) exhibited spurts of movement interrupted by pauses. The retrogradely moving $\mathrm{NF}$ (NF 8, filled squares) first entered the field at $\approx 205 \mathrm{sec}$ into the sequence and moved in a relatively sustained manner until the end of the sequence. Of the other NFs, three spent the entire time paused, exhibiting no movements at all (NFs 3, 6, and 7). The remaining three NFs (1, 2, and 4) spent most of their time paused but exhibited occasional spurts of movements that spanned 5-10 sec. As a measure of total NF transport in this sequence, we pooled all data for the distance moved per $5 \mathrm{sec}$ interval for all of the NFs that were examined, and then we determined what proportion of the data points met our criteria for movement $(\geq 0.3 \mu \mathrm{m}$ in $5 \mathrm{sec})$. This provided an estimate for the fraction of time that the NF population in this axon segment spent moving. The cumulative time in which that population of NFs moved accounted for only $20 \%$ of the total time that it was observed. During the remaining $80 \%$ of the observation time these NFs were paused.

The number of NFs that exhibited sustained movement in this sequence was typical of other sequences spanning similar time periods. The number of NFs undergoing sustained movement were counted in 16 extended sequences from this study. These sequences spanned time periods of 5-20 min, and the number of moving NFs detected in each sequence ranged from two to six, with an average of four. Thus, on average, one NF underwent sustained movement for every $3.6 \mathrm{~min}$ of sequence; similar results were reported by Wang et al. (2000). The sequence used for the above analysis comparing time spent paused versus moving spanned $\approx 5 \mathrm{~min}$, and during this time two NFs moved in a sustained manner. Thus, this sequence is representative with regard to the number of NFs exhibiting sustained transport, suggesting that our estimate for the fraction of time that the NF population in this axon spent moving is representative of NF behaviors seen in the total population of axons that were studied. In aggregate, the NFs in axon segments spend the vast majority of time paused in their transit within the axon.

\section{DISCUSSION}

Using an experimental model for visualizing individual NFs in living axons, we observed NFs tagged with a GFP-NFH chimera to undergo rapid vectorial movement in living axons. Transport of NFs was highly asynchronous, which presumably reflects events that operate stochastically on individual NFs. A long-standing controversy regarding axonal transport of NF proteins concerns whether they are transported as NFs or in a nonfilamentous form (for review, see Baas and Brown, 1997; Hirokawa et al., 1997). The present results, together with similar findings obtained independently by Wang et al. (2000), establish unequivocally that NFs themselves are conveyed in axonal transport and provide insights into possible mechanisms for the axonal transport of cytoskeletal structures.

Before discussing our findings, we note that Yabe et al. (1999) reported very different results using a GFP-NFM construct to examine NF transport. After transfection the GFP-NFM chimera did not assemble into NFs but instead appeared in dot-like structures that underwent a slow jiggling motion. These results differ significantly from ours and those of Wang et al. (2000) in two major respects. First, in these latter studies most of the transfected protein assembled into NFs, and little or none was seen in dot-like structures. Second, the movements of NFs were rapid and clearly vectorial in nature. We suspect that differences in the GFP-NF protein construct account for the different results obtained by Yabe et al. (1999) as compared with our studies. In our studies and several others (Ho et al., 1998; Yoon et al., 1998; Wang et al., 2000) GFP-intermediate filament proteins have been prepared that assemble into intermediate filaments. In these studies GFP was ligated to the $\mathrm{N}$ terminus of the intermediate filament protein. In Yabe et al. (1999), GFP was ligated to the C terminus of NFM. Liem has prepared GFP-NFM constructs in which GFP was ligated to the $\mathrm{C}$ terminus. The resulting GFP-NFM was not only assemblyincompetent but disrupted endogenous filaments when it was transfected into cells (R. K. H. Liem, unpublished data). To generate assembly-competent GFP-NFM, we found that it was necessary to ligate GFP at the N terminus of NFM. Thus, we suspect that the construct used by Yabe et al. (1999) was assembly-incompetent and therefore that their results may not reflect normal NF behavior.

The studies of Yabe et al. (1999) are part of a literature that proposes that NF proteins are transported in a nonfilamentous form (for review, see Nixon, 1998). In many of these studies the essential observation is that some of the axonal NF protein is soluble in nonionic detergents (Tsuda et al., 1997; Jung et al., 1998). However, the relationship of detergent solubility to the in vivo organization and transport form of NF proteins is at best indirect. In an apparently more direct approach to test this hypothesis, Terada et al. (1996) used mice expressing a LacZ-NFH transgene in which NFs are aggregated in the cell body and so most axons are NF-free. Dorsal root ganglion neurons of transgenic mice were transfected to express epitope-tagged NFM, and its distribution was determined at varying times thereafter. Some of the epitopetagged NFM was seen in axons. Because the axons generally had no detectable NFs, the authors suggested that the tagged NFM was transported in a nonfilamentous form and that this reflected normal slow transport of NFM. Although this is one interpretation, it is also possible that the appearance of the tagged NFM in axons reflects transport, but not normal NFM transport. Overexpressing extra NFM in neurons in which NF protein organization is already grossly abnormal may result in nonphysiological interactions of the extra NFM with other proteins that are transported, and through such abnormal interactions NFM may be carried into the axon.

In our view the proposal that NF proteins are transported in a nonfilamentous form, although a formal possibility, is not well supported by the available data. Our studies and those of Wang et al. (2000) do not address directly whether NF proteins are transported in a nonfilamentous form. However, they do demonstrate unambiguously that NFs themselves undergo vigorous vectorial transport in axons, and, as discussed below, the character of this transport is sufficient to explain the NF protein transport behavior described in classic studies on slow axonal transport.

The present results and those of Wang et al. (2000) significantly refine the current understanding of slow axonal transport because the behavior of individual NFs in living axons was observed for the first time. Surprisingly, single NFs underwent spurts of relatively rapid movements alternating with periods having little or no movement. Three key characteristics of NF transport emerge from these data. First, during spurts of movement the NFs moved at a broad spectrum of rates, ranging from $\leq 0.1 \mu \mathrm{m} / \mathrm{sec}$ to nearly $1.7 \mu \mathrm{m} / \mathrm{sec}$, with an average of $\approx 0.6 \mu \mathrm{m} / \mathrm{sec}$. Second, the transport of NFs was highly asynchronous, in that in any given axon over any given time period some NFs moved whereas others did not, and NFs moving in the same region of an axon often exhibited very different behaviors. Finally, at any given moment most NFs are paused in their transit in the axon, with only a small percentage actually moving. 
Wang et al. (2000) reported that NFs spent minimally $\approx 73 \%$ of the time paused. Using a different strategy in which several NFs were tracked in a single axon over an extended time, we found that, as a group, the NFs spent $\approx 20 \%$ of their time moving and $\approx 80 \%$ paused. Although these numbers derive from one axon, the conclusion that NFs generally spend much more time paused than moving is consistent with observations that most NFs detectable in all sequences move little or not at all. Although many NFs were present in our sequences, on average we detected only one NF to undergo sustained movement for every $3.6 \mathrm{~min}$ of observation. Collectively, these observations indicate that transport events occur relatively infrequently.

Although NFs spend most of their time paused, these pauses appear to be transient, and the data suggest that paused NFs will resume transport if they are observed long enough. For example, many NFs started moving after extended pauses (see Fig. 8). Moreover, time lapse sequences that used 10-20 min sampling intervals for $\geq 60 \mathrm{~min}$ found that the distribution of tagged NFs changed (data not shown). However, the sequences were conf using because few NFs could be tracked unambiguously at these sampling rates. Data on NF transport with short (5 sec) sampling intervals indicate that many NFs must have moved in experiments that used long sampling intervals, but poor temporal resolution precluded detailed analyses. This pattern of NF transport is analogous to the transport of mitochondria, which show frequent and often extended pauses during their transit in axons. As a result, over relatively short time intervals only a portion of the mitochondria moves, whereas the entire population moves with more extended observation times (Martz et al., 1984; Morris and Hollenbeck, 1995). The basis for pauses in NF transport is unknown. In this regard, many morphological studies have revealed side arms that extend from NFs that may interconnect NFs transiently with each other and with other structures (Mulligan et al., 1991; Nixon, 1998). Such transient interactions may contribute to pauses in NF transport.

Collectively, our observations plus those of Wang et al. (2000) provide the following picture of NF transport. NFs undergo vectorial movement in axons. This movement is sporadic, with individual NFs displaying periods of sustained movement interspersed with intervals of no movement. Spurts of movements occur in both anterograde and retrograde directions but with a strong anterograde bias. Furthermore, movement of a given NF is not obviously coordinated with that of other NFs. In general, individual NFs spend more time paused than moving, and, as a result, movements were infrequent during the 5-20 min periods of observation. However, most if not all NFs undergo transport over more extended times so that the population of NFs advances within the axon toward the axon tip. The rate of advance for the NF population represents an average of distances moved during spurts of transport and the absence of movement during pauses. We can estimate this rate as follows. If individual NFs have a net anterograde transport of $0.3 \mu \mathrm{m} / \mathrm{sec}$ when moving and they move $20 \%$ of the time, then the population of NFs will advance down the axon at $0.06 \mu \mathrm{m} / \mathrm{sec}$, on average. This value is somewhat faster than the $\approx 1 \mathrm{~mm} / \mathrm{d}$ $(\approx 0.01 \mu \mathrm{m} / \mathrm{sec})$ obtained from pulse-labeling studies of NF protein transport in mature neurons (Lasek et al., 1992), but it agrees well with rates of $3-8 \mathrm{~mm} / \mathrm{d}(0.03-0.09 \mu \mathrm{m} / \mathrm{sec})$ measured in developing neurons (Willard and Simon, 1983; Koehnle and Brown, 1999).

Previous attempts to visualize NF transport in cultured neurons by using photobleaching approaches did not detect NF movement. These data were used to argue that NFs are not transported in axons (Okabe et al., 1993; Nixon, 1998). These experiments were designed to test the specific hypothesis that the NF population moved continuously and unidirectionally in the axon at slow transport rates, as determined in pulse-labeling studies $(0.01 \mu \mathrm{m} / \mathrm{sec})$. Thus, photobleaching was used to mark a population of NFs, and relatively large time compressions (intervals between images of several minutes or longer) were used in time lapse video microscopy to determine whether the population of tagged NFs moved anterogradely at slow transport rates (Okabe et al., 1993). The results showed that the tagged NFs did not behave according to this model. However, the failure to detect movement in these experiments does not justify the conclusion that NFs do not move at all, because the experiments were designed to reveal movement of a specific character. By observing individual NFs, we and Wang et al. (2000) demonstrated that NFs are translocated in axons, and we confirm that this movement does not conform to the model of coherent movement of the NF population. Instead, individual NFs move independently of each other; for any individual NF the movement events are relatively rare, but when NFs move, they move rapidly. Photobleaching or other strategies that provide information on populations of NFs are not well suited for the detection of these types of movements, especially when long time compressions are used.

The original proposal that NFs are transported in axons was part of a broader structural perspective of axonal transport, which hypothesized that the cytoskeletal proteins tubulin, actin, and NF proteins are transported in axons in their respective polymeric form (for review, see Baas and Brown, 1997). This polymer transport hypothesis has been controversial because of difficulties in identifying the transport form of these proteins. This controversy has been resolved at least partially with the direct demonstration of NF transport in living axons. Nonetheless, the transport of microtubules (MTs) and actin filament has not been demonstrated directly, and, in fact, many reports have argued that MTs and actin filaments are not transported. Most of these studies used photobleaching or photoactivation approaches and generally detected no movement of the population of marked polymers (Lim et al., 1990; Okabe and Hirokawa, 1990; Sabry et al., 1995; Takeda et al., 1995; Chang et al., 1998). However, if MTs and actin filaments are transported in an intermittent and asynchronous manner like NFs, then the failure of these approaches to detect polymer transport may be a consequence of the experimental design rather than a lack of movement. A more conservative interpretation of these studies is that MTs (and actin filaments) are not transported en masse within the axon.

Recently, Chang et al. (1999) used a novel application of digital fluorescence microscopy that permitted observation of individual MTs in axons of cultured frog neurons. They did not detect vectorial movement of MTs and concluded that MTs are not transported in axons. These studies used observation times of 3-10 min. We have many time lapse sequences spanning 5-10 min or more in which no NF movement was apparent, and similar results were reported by Wang et al. (2000). Typically, only one NF moved for every $\approx 3.6 \mathrm{~min}$ of observation. If this frequency of movement applies to MTs, then Chang et al. (1999) may have missed MT transport events because of their relatively short observation times. This problem is exacerbated further by the high MT density in axons, which restricted their observations to a subset of MTs. Although the issue of MT transport in axons has not been resolved definitively, our bias is that such transport occurs. This derives from studies that (1) provided indirect evidence that MTs move in axons (Baas and Brown, 1997; Slaughter et al., 1997) and (2) revealed that mechanisms exist in cultured neurons to translocate MTs (Ahmad et al., 1998; Baas, 1999; Dent et al., 1999). Finally, the demonstration of NF transport in axons provides strong precedent for the validity of the polymer transport model.

The mechanism of NF transport is a matter of speculation. The maximum rates of $\mathrm{NF}$ transport are comparable to those of membrane-bounded organelles along MTs (Allen et al., 1982; Brady et al., 1985). Indeed, these maximum rates are consistent with rates produced by microtubule-based motors of the cytoplasmic dynein and kinesin families (Brady and Sperry, 1995). This raises the possibility that NF transport is powered by the same type of motors that power vesicle transport (Brady, 2000).

Several models for NF transport in axons are possible. One is that NFs are cargo moved by MT motors along MTs, with plusend-directed motors moving NFs anterogradely and minus-enddirected motors moving NFs retrogradely. Another model is that NFs move by piggy-backing along MTs. In this view the NFs are 
not moved directly by motors but instead move via association with transported MTs. In this regard, ample precedent exists for MT-NF interactions in axons (Aamodt and Williams, 1984; Heimann et al., 1985; Dalpe et al., 1998; Yang et al., 1999; Herrmann and Aebi, 2000). Also, MTs and NFs are cotransported in pulse label studies (Brady and Lasek, 1982), further supporting linkage of their transport. Finally, MTs can move anterogradely and retrogradely in axons (Dent et al., 1999). Thus, the directionality of MT movements can account for both anterograde and retrograde movements of NFs. Cytoplasmic dynein and dynactin have been implicated in MT transport in axons (Dillman et al., 1996a,b; Ahmad et al., 1998; Baas, 1999). Because dynein can translocate MTs with their plus-ends or minus-ends leading, a single motor can account for the bidirectional transport of MTs. If NFs move by association with transported MTs, then dynein also can mediate the anterograde and retrograde movements of NFs. The close similarity in transport rates of anterogradely and retrogradely moving NFs is consistent with the possibility of a single motor mediating the movement in both directions. We cannot distinguish between these or other possibilities on the basis of existing data. However, the availability of model systems for visualizing NF transport directly provides the opportunity to dissect the mechanisms that transport cytoskeletal polymers in axons. These mechanisms contribute to neuronal morphogenesis by providing the axon with the architectural elements required to generate and maintain its elongate shape and also by generating forces within the axon and axon tip that are involved in axon growth and navigation (Ahmad et al., 2000). With the development of these model systems for visualizing cytoskeletal transport in growing axons, we now can begin to explore how cytoskeletal transport and transport motors contribute to these fundamental aspects of neuronal morphogenesis.

\section{REFERENCES}

Aamodt EJ, Williams Jr RC (1984) Microtubule-associated proteins connect microtubules and neurofilaments in vitro. Biochemistry 23:6023-6031. Ahmad FJ, Baas PW (1995) Microtubules released from the neuronal centrosome are transported into the axon. J Cell Sci 108:2761-2769.

Ahmad FJ, Joshi HC, Centonze VE, Baas PW (1994) Inhibition of microtubule nucleation at the neuronal centrosome compromises axon growth. Neuron 12:271-280.

Ahmad FJ, Echeverri CJ, Vallee RB, Baas PW (1998) Cytoplasmic dynein and dynactin are required for the transport of microtubules into the axon. J Cell Biol 140:391-401.

Ahmad FJ, Hughey J, Wittmann T, Hyman A, Greaser M, Baas PW (2000) Motor proteins regulate force interactions between microtubules and microfilaments in the axon. Nat Cell Biol 2:267-280.

Allen RD, Metuzals J, Tasaki I, Brady ST, Gilbert SP (1982) Fast axonal transport in squid giant axon. Science 218:1127-1129.

Baas PW (1999) Microtubules and neuronal polarity: lessons from mitosis. Neuron 22:23-31.

Baas PW, Ahmad FJ (1993) The transport properties of axonal microtubules establish their polarity orientation. J Cell Biol 120:1427-1437.

Baas PW, Brown A (1997) Slow axonal transport: the polymer transport model. Trends Cell Biol 7:380-384.

Black MM, Lasek RJ (1980) Slow components of axonal transport: two cytoskeletal networks. J Cell Biol 86:616-623.

Black MM, Keyser P, Sobel E (1986) Interval between the synthesis and assembly of cytoskeletal proteins in cultured neurons. J Neurosci 6:1004-1012.

Black MM, Slaughter T, Moshiach S, Obrocka M, Fischer I (1996) Tau is enriched on dynamic microtubules in the distal region of growing axons. J Neurosci 16:3601-3619.

Brady ST 2000 Neurofilaments run sprints, not marathons. Nat Cell Biol 2:E43-E45.

Brady ST, Lasek RJ (1982) The slow components of axonal transport: movements, compositions, and organization. In: Axoplasmic transport (Weiss D, ed), pp 206-217. New York: Springer.

Brady ST, Sperry AO (1995) Biochemical and functional diversity of microtubule motors in the nervous system. Curr Opin Neurobiol 5:551-558.

Brady ST, Lasek RJ, Allen RD (1985) Video microscopy of fast axonal transport in isolated axoplasm: a new model for study of molecular mechanisms. Cell Motil 5:81-101.

Brown A (1997) Visualization of single neurofilaments by immunofluorescence microscopy of splayed axonal cytoskeletons. Cell Motil Cytoskeleton 38:133-145.

Brown A, Slaughter T, Black MM (1992) Newly assembled microtubules are concentrated in the proximal and distal regions of growing axons. J Cell Biol 119:867-882.
Chang S, Rodionov VI, Borisy GG, Popov SV (1998) Transport and turnover of microtubules in frog neurons depend on the pattern of axonal growth. J Neurosci 18:821-829.

Chang S, Svitkina TM, Borisy GG, Popov SV (1999) Speckle microscopic evaluation of microtubule transport in growing nerve processes. Nat Cell Biol 1:399-403.

Chin SS, Liem RK (1990) Transfected rat high-molecular-weight neurofilament (NF-H) coassembles with vimentin in a predominantly nonphosphorylated form. J Neurosci 10:3714-3726.

Ching GY, Liem RK (1993) Assembly of type IV neuronal intermediate filaments in non-neuronal cells in the absence of preexisting cytoplasmic intermediate filaments. J Cell Biol 122:1323-1335.

Chun LL, Patterson PH (1977) Role of nerve growth factor in the development of rat sympathetic neurons in vitro. I. Survival, growth, and differentiation of catecholamine production. J Cell Biol 75:694-704.

Dalpe G, Leclerc N, Vallee A, Messer A, Mathieu M, De Repentigny Y, Kothary R (1998) Dystonin is essential for maintaining neuronal cytoskeleton organization. Mol Cell Neurosci 10:243-257.

Dent EW, Callaway JL, Szebenyi G, Baas PW, Kalil K (1999) Reorganization and movement of microtubules in axonal growth cones and developing interstitial branches. J Neurosci 19:8894-8908.

Dillman JF, Dabney LP, Karki S, Paschal BM, Holzbaur EL, Pfister KK (1996a) Functional analysis of dynactin and cytoplasmic dynein in slow axonal transport. J Neurosci 16:6742-6752.

Dillman JF, Dabney LP, Pfister KK (1996b) Cytoplasmic dynein is associated with slow axonal transport. Proc Natl Acad Sci USA 93:141-144.

Glass JD, Griffin JW (1994) Retrograde transport of radiolabeled cytoskeletal proteins in transected nerves. J Neurosci 14:3915-3921.

Graham FL, Prevec L (1991) Manipulation of adenovirus vectors. In: Methods in molecular biology: gene transfer and expression protocols (Murray EJ, ed), pp 109-127. Clifton, NJ: Humana.

Heimann R, Shelanski ML, Liem RK (1985) Microtubule-associated proteins bind specifically to the $70 \mathrm{kDa}$ neurofilament protein. J Biol Chem 260:12160-12166.

Herrmann H, Aebi U 2000 Intermediate filaments and their associates: multitalented structural elements specifying cytoarchitecture and cytodynamics. Curr Opin Cell Biol 12:79-90.

Hirokawa N, Terada S, Funakoshi T, Takeda S (1997) Slow axonal transport: the subunit transport model. Trends Cell Biol 7:384-388.

Ho CL, Martys JL, Mikhailov A, Gundersen GG, Liem RKH (1998) Novel features of intermediate filament dynamics revealed by green fluorescent protein chimeras. J Cell Sci 111:1767-1778.

Hollenbeck PJ (1993) Products of endocytosis and autophagy are retrieved from axons by regulated retrograde organelle transport. J Cell Biol 121:305-315.

Hollenbeck PJ, Bray D (1987) Rapidly transported organelles containing membrane and cytoskeletal components: their relation to axonal growth. J Cell Biol 105:2827-2835.

Jung C, Yabe J, Wang F-S, Shea TB (1998) Neurofilament subunits can undergo axonal transport without incorporation into Triton-insoluble structures. Cell Motil Cytoskeleton 40:44-58.

Koehnle TJ, Brown A (1999) Slow axonal transport of neurofilament protein in cultured neurons. J Cell Biol 144:447-458.

Lasek RJ (1980) The dynamics of neuronal structures. Trends Neurosci 3:87-91.

Lasek RJ, Hoffman PN (1976) Section 8: Cilia, flagella, axostyles and neurons. In: Cell motility (Goldman R, Pollard T, Rosenbaum J, eds), pp 1021-1049. Cold Spring Harbor, NY: Cold Spring Harbor Laboratory.

Lasek RJ, Paggi P, Katz MJ (1992) Slow axonal transport mechanisms move neurofilaments relentlessly in mouse optic axons. J Cell Biol 117:607-616.

Lasek RJ, Paggi P, Katz MJ (1993) The maximum rate of neurofilament transport in axons: a view of molecular transport mechanisms continuously engaged. Brain Res 616:58-64.

Lee MK, Xu Z, Wong PC, Cleveland DW (1993) Neurofilaments are obligate heteropolymers in vivo. J Cell Biol 122:1337-1350.

Li Y, Black MM (1996) Microtubule assembly and turnover in growing axons. J Neurosci 16:531-544.

Lim S-S, Edson KJ, Letourneau PC, Borisy GG (1990) A test of microtubule translocation during neurite elongation. J Cell Biol 111:123-130.

Martz D, Lasek RJ, Brady ST, Allen RD (1984) Mitochondrial motility in axons: membranous organelles may interact with the force generating system through multiple surface binding sites. Cell Motil 4:89-102.

Mobley WC, Shenker A, Shooter EM (1976) Characterization and isolation of proteolytically modified nerve growth factor. Biochemistry 15:5543-5551.

Morris RL, Hollenbeck PJ (1995) Axonal transport of mitochondria along microtubules and F-actin in living vertebrate neurons. J Cell Biol 131:1315-1326.

Mulligan L, Balin BJ, Lee VM, Ip W (1991) Antibody labeling of bovine neurofilaments: implications on the structure of neurofilament side arms. J Struct Biol 106:145-160.

Nixon RA (1998) The slow axonal transport of cytoskeletal proteins. Curr Opin Cell Biol 10:87-92.

Okabe S, Hirokawa N (1990) Turnover of fluorescently labeled tubulin and actin in the axon. Nature 343:479-482. 
Okabe S, Miyasaka H, Hirokawa N (1993) Dynamics of the neuronal intermediate filaments. J Cell Biol 121:375-386.

Sabry J, O’Connor TP, Kirschner MW (1995) Axonal transport of tubulin in Ti1 pioneer neurons in situ. Neuron 14:1247-1256.

Schliwa M, van Blerkom JJ (1981) Structural interactions of cytoskeleta components. J Cell Biol 90:222-235.

Slaughter T, Wang J, Black MM (1997) Microtubule transport from the cell body into the axons of growing neurons. J Neurosci 17:5807-5819.

Smith GM, Hale J, Panikowski EM, Lindsay R, Wong V, Rudge JS (1996) Astrocytes infected with replication-defective adenovirus containing a secretory form of CNTF or NT-3 show enhanced support of neuronal populations in vitro. Exp Neurol 139:156-166.

Takeda S, Funakoshi T, Hirokawa N (1995) Tubulin dynamics in neuronal axons of living zebrafish embryos. Neuron 14:1257-1264.

Terada S, Nakata T, Peterson AC, Hirokawa N (1996) Visualization of slow axonal transport in vivo. Science 273:784-788.

Tsuda M, Tashiro T, Komiya Y (1997) Increased solubility of highmolecular-mass neurofilament subunit by suppression of dephosphorylation: its relation to axonal transport. J Neurochem 68:2558-2565.
Tytell M, Black MM, Garner JA, Lasek RJ (1981) Axonal transport: each major rate component reflects the movement of distinct macromolecular complexes. Science 214:179-181.

Wang L, Ho C-L, Sun D, Liem RKH, Brown A (2000) Rapid movements of neurofilaments interrupted by prolonged pauses. Nat Cell Biol 2:137-141.

Willard M, Simon C (1983) Modulations of neurofilament axonal transport during the development of rabbit retinal ganglion cells. Cell 35:551-559.

Yabe JT, Pimenta A, Shea TB (1999) Kinesin-mediated transport of neurofilament protein oligomers in growing axons. J Cell Sci 112:3799-3814

Yang Y, Bauer C, Strasser G, Wollman R, Julien JP, Fuchs E (1999) Integrators of the cytoskeleton that stabilize microtubules. Cell 98:229-238.

Yoon M, Moir RD, Prahlad V, Goldman RD (1998) Motile properties of vimentin intermediate filament networks in living cells. J Cell Biol 143:147-157.

Yu W, Schwei MJ, Baas PW (1996) Microtubule transport and assembly during axon growth. J Cell Biol 133:151-157.

Zhang WW, Fang X, Branch CD, Mazur W, French BA, Rothe JA (1993) Generation and identification of recombinant adenovirus by liposomemediated transfection and PCR analysis. Biotechniques 15:868-872. 